\title{
Development of shear locking-free shell elements using an enhanced assumed strain formulation
}

\author{
José M. A. César de Sá*, ${ }^{*}$ Renato M. Natal Jorge $\ddagger$, \\ Robertt A. Fontes Valente ${ }^{\S}$ and Pedro M. Almeida Areias \\ Department of Mechanical Engineering, Faculty of Engineering, University of Porto, \\ Rua Dr. Roberto Frias, 4200-465, Porto, Portugal
}

\begin{abstract}
SUMMARY
The degenerated approach for shell elements of Ahmad and co-workers is revisited in this paper. To avoid transverse shear locking effects in four-node bilinear elements, an alternative formulation based on the enhanced assumed strain (EAS) method of Simo and Rifai is proposed directed towards the transverse shear terms of the strain field. In the first part of the work the analysis of the null transverse shear strain subspace for the degenerated element and also for the selective reduced integration (SRI) and assumed natural strain (ANS) formulations is carried out. Locking effects are then justified by the inability of the null transverse shear strain subspace, implicitly defined by a given finite element, to properly reproduce the required displacement patterns. Illustrating the proposed approach, a remarkably simple single-element test is described where ANS formulation fails to converge to the correct results, being characterized by the same performance as the degenerated shell element. The adequate enhancement of the null transverse shear strain subspace is provided by the EAS method, enforcing Kirchhoff hypothesis for low thickness values and leading to a framework for the development of shear-lockingfree shell elements. Numerical linear elastic tests show improved results obtained with the proposed formulation. Copyright (c) 2001 John Wiley \& Sons, Ltd.
\end{abstract}

KEY WORDS: shell elements; enhanced assumed strain method; transverse shear locking; enhanced transverse shear strain field

\section{INTRODUCTION}

In the finite element analysis of shell structures, a landmark can be established with the work of Ahmad et al. [1] leading to the so-called degenerated shell element concept. This

\footnotetext{
*Correspondence to: José M. A. Cesar de Sá, Department of Mechanical Engineering, Faculty of Engineering, University of Porto, Rue Dr. Roberto Frias, 4200-465, Porto, Portugal

$\dagger$ E-mail: cesarsa@fe.up.pt

‡E-mail: rnatal@fe.up.pt

E-mail: robertt@fe.up.pt

Contract/grant sponsor: MCT-FCT Portugal; contract/grant numbers: PRAXIS XXI/BD/21662/99 and PRAXIS $\mathrm{XXI} / \mathrm{BD} / 18533 / 98$

Contract/grant sponsor: FEDER; contract/grant number: POCTI/33640/EME/2000

Copyright (c) 2001 John Wiley \& Sons, Ltd.

Received 2 October 2000

Revised 5 March 2001
} 
formulation is obtained by judiciously imposing some chosen kinematic and mechanical assumptions on the three-dimensional continuum, circumventing the use of classical thin shell theories [2,3]. In Reference [2] Bucalem and Bathe highlighted as some key features of this class of elements the applicability to any shell geometry, the use of $C^{0}$ conforming displacement-based elements and the adoption of 'engineering' degrees of freedom such as displacements and rotations.

However, it is well known that degenerated formulations possess strong deficiencies in reproducing the behaviour of thin shell structures, leading to locking phenomena. For lowerorder elements, like the four-node bilinear element based on a displacement formulation, these effects are responsible for the complete deterioration of the results for thin shells.

The shear locking, affecting these elements, can be traced to the fact that no KirchhoffLove hypothesis is introduced into the displacement interpolations. The transverse shear strains cannot then vanish at all points in the element when it is subjected to a pure bending state $[4,5]$.

Amongst the approaches to overcome this problem, the simplest and earliest of all was the use of uniform or selective reduced integration, as firstly reported in References [6, 7]. In general [2], the uniform reduced integration (URI) leads to spurious zero energy modes although in some cases a correct solution is obtained. For the selective reduced integration (SRI) the same problems are posed, but frequently to a smaller degree. In the specific case of plate elements, the work of Belytschko and Tsay [8] pioneered a range of contributions using stabilization procedures for controlling the kinematics modes.

Other techniques were proposed to alleviate the excess of stiffness characterizing locking patterns, most of them being classified as hybrid, mixed or hybrid-mixed methods. For these formulations one or more independent field assumptions for strains, stress and/or incompatible displacements can be assumed, in conjunction with the usual displacement approximation. The additional fields may be discontinuous from element to element so that the corresponding unknowns can be eliminated at the element level $[5,9]$. These methods include, among others, procedures such as the assumed natural strain (ANS) approach (for bilinear shell elements, see References [10-12]; for higher-order elements see, for example, References [13-15]) and the enhanced assumed strain (EAS) formulation [5, 16-19].

The key idea of the ANS method is the replacement, in the minimum potential energy principle, of selected displacement-related strains by independently assumed strain fields in element natural co-ordinates [20]. The variational basis can be found, for example, in the work of Militello and Felippa [9] and the performance of the elements derived from this formulation relies strongly in choosing correctly the sample strain positions for the assumed interpolation. In addition, some deficiencies in the case of irregular meshes can be pointed out [5]. Nevertheless, the bilinear MITC4 element of Dvorkin and Bathe [10], falling in that category, is amongst the most employed shell formulations, "which sometimes cause difficulties for other assumed strain techniques' [21].

The EAS method, as firstly proposed by Simo and Rifai [16], uses a three-field mixed functional in terms of displacements, stresses and an enhanced strain field, based on the $\mathrm{Hu}-$ Washizu variational principle. As discussed in this reference, the total strain field is built up as a summation of the (compatible) symmetric gradient of the displacement field and the enhanced strain. This latter is not subjected to any interelement continuity requirement, and can be related to an 'incompatible mode' field. Providing a general formulation, Simo and Rifai presented a bilinear isoparametric plate element with enhanced transverse 
shear strain field. For a square configuration this element proved to be identical to MITC4 element [16].

A deeper application of the enhanced techniques in shell analysis came shortly after with the work of Andelfinger and Ramm [5] in the linear elastic range. The extension to non-linear aspects was carried out in subsequent publications (see, for instance, References $[17,18,22,23])$.

Relating the low performance of MITC4 in the presence of distorted meshes to in-plane deficiencies, Andelfinger and Ramm developed an enhanced membrane and bending strain field composed of seven internal parameters. Despite the improvements obtained, their procedures were not extended to shear strain terms, as they kept the assumed natural strain approach of MITC4 in order to attenuate transverse shear locking effects.

Within a different philosophy, the present work develops an approach suitable for the treatment of the transverse shear locking phenomenon, relying on the enhanced assumed strain formulation and applied over the degenerated four-node element.

Following the methodology of the original work of César de Sá and Owen [24] and, subsequently, César de Sá and Natal Jorge [25] for two-dimensional incompressible problems, shear locking appearance is related to the inability of a basis of the null transverse shear strain subspace, implicitly defined by each element formulation, to accurately reproduce the required deformation patterns.

A deep analysis of MITC4 and selective integrated (SRI) formulations, leading to possible bases for their null transverse shear strain subspaces, reveals the missing terms on the respective basis for the degenerated element. Performing an enhancement over the covariant shear strain terms of this element, an enlargement of its subspace is obtained, leading to a new class of degenerated shell elements with improved behaviour.

The specific analysis of the null transverse shear strain subspace carried out in this work also revealed that MITC4 element lacks two missing components in its basis, when compared to SRI elements. This same component is not present in the basis of the degenerated element. In fact, in trying to reproduce the deformation pattern corresponding to this absent component, both elements reveal a strong deterioration of results. In this specific, single element, example MITC4 and Ahmad et al. formulations provide the same numerical result, while the SRI approach shows no shear locking.

It is worth noting the important insight over locking phenomena brought forward by the work of Pitkäranta et al. [26] and Chapelle and Bathe [27]. Based on a detailed analysis of bending and membrane subspaces, those authors developed a class of benchmark tests for the behaviour characterization of shell elements, which will be used in this paper (for details see References [28-30]).

An outline of the paper is as follows. In Section 2, the analysis of the null transverse shear strain subspace implicitly defined for each finite element formulation is showed. Three architectures of shell finite elements are described: the original degenerated approach (applied to the bilinear four-node element), the ANS formulation for MITC4 element and the SRI method. Different subspace dimensions are then obtained for each element, justifying the different performances of each formulation in dealing with transverse shear locking phenomena. Based on these results, in Section 3 suitable enhanced interpolation matrices are proposed, attenuating shear locking effects for thin shells. Acting over the four-node degenerated element a class of new elements, with an enhanced transverse shear strain field is presented. Following an alternative track a different proposal relying on the enhancement of the MITC4 element 
and grouping EAS and ANS techniques is proposed. Finally, Section 4 provides numerical test problems stating the performance of the presented elements when compared to previous published results for well-known shell elements available in the literature.

\section{BASES FOR THE SUBSPACE OF NULL TRANSVERSE SHEAR STRAINS}

\subsection{Introduction}

In the analysis of shear locking phenomena, attention is focused on the transverse components of the strain field, defined in the natural set of co-ordinates $(\xi, \eta, \zeta)$. Related to this system, the displacement dependent strain field for a given point within an element can be represented in a discrete vector form as

$$
\mathbf{E} \equiv \mathbf{E}^{\mathrm{u}}=\left\{\begin{array}{llllll}
E_{\xi \xi}^{\mathrm{u}} & E_{\eta \eta}^{\mathrm{u}} & E_{\zeta \zeta}^{\mathrm{u}} & E_{\zeta \eta}^{\mathrm{u}} & E_{\xi \zeta}^{\mathrm{u}} & E_{\eta \zeta}^{\mathrm{u}}
\end{array}\right\}^{\mathrm{T}} \equiv \mathbf{Q} \mathbf{d}_{n}
$$

being $\mathbf{Q}$ a matrix grouping differential terms, and relating nodal displacements $\mathbf{d}_{n}$ with strains.

According to the usual representation of degenerated elements $[1,31]$, the nodal displacement vector includes three translational degrees of freedom (defined in the global co-ordinate system) and two independent rotational degrees of freedom (defined in a local frame) as

$$
\mathbf{d}_{n}=\left.\left\{\begin{array}{lllll}
u & v & w & \theta_{1} & \theta_{2}
\end{array}\right\}\right|_{n} ^{\mathrm{T}}
$$

for a given node $(n)$.

The natural strain components described in Equation (1) can be evaluated, for the linear case [31], from the equation

$$
\mathbf{E}_{\xi_{i} \xi_{j}}^{\mathrm{u}}=\frac{1}{2}\left(\frac{\partial \mathbf{u}}{\partial \xi_{i}} \cdot \mathbf{g}_{j}+\frac{\partial \mathbf{u}}{\partial \xi_{j}} \cdot \mathbf{g}_{i}\right) \quad \text { for }\left(i, j=1,2,3 ; \xi_{1}=\xi, \quad \xi_{2}=\eta, \quad \xi_{3}=\zeta\right)
$$

based on the displacement vector $\mathbf{u}$ and the covariant base vectors

$$
\mathbf{g}_{i}=\frac{\partial \mathbf{x}}{\partial \xi_{i}}
$$

with $\mathbf{x}$ being the position vector.

The importance of the described natural strain field is related to the imposition of the Kirchhoff constraints for thin shells, requiring that those strains should vanish as thickness values tends to zero. When applying the Mindlin-type hypothesis, this condition cannot be satisfied at all points over a specified domain. However, in an 'average' sense [32] this can be accomplished as follows:

$$
\begin{aligned}
& \int_{\Delta A} E_{\xi \zeta}^{\mathrm{u}} \mathrm{d} \xi \mathrm{d} \eta=0 \\
& \int_{\Delta A} E_{\eta \zeta}^{\mathrm{u}} \mathrm{d} \xi \mathrm{d} \eta=0
\end{aligned}
$$

where $\Delta A$ represents the particular area analysed. 
The assumption that the shear strain field $E_{\xi \zeta}^{\mathrm{u}}$ is a linear function of $\xi$ and, in a similar way, $E_{\eta \zeta}^{\mathrm{u}}$ is a linear function of $\eta[32]$ leads to the equations

$$
\begin{aligned}
& \int_{-1}^{0} E_{\zeta \zeta}^{\mathrm{u}} \mathrm{d} \xi=0, \quad \int_{0}^{+1} E_{\xi \zeta}^{\mathrm{u}} \mathrm{d} \xi=0 \\
& \int_{-1}^{0} E_{\eta \zeta}^{\mathrm{u}} \mathrm{d} \eta=0, \quad \int_{0}^{+1} E_{\eta \zeta}^{\mathrm{u}} \mathrm{d} \eta=0
\end{aligned}
$$

The imposition of these constraints discretely at the Gauss points leads to

$$
\left\{\begin{array}{l}
\mathbf{E}_{\xi \zeta} \\
\mathbf{E}_{\eta \zeta}
\end{array}\right\}=\mathbf{Q}_{\text {shear }} \mathbf{d}_{\mathrm{e}}=\mathbf{0}
$$

with $\mathbf{d}_{\mathrm{e}}$ encompassing the nodal degrees of freedom within a specified element. Following previous works of the authors $[24,25,33]$ it is possible to define

$$
I_{\mathrm{h}}^{\mathrm{s}}=\left\{\mathbf{u}_{\mathrm{h}} \in U_{\mathrm{h}}: \mathbf{Q}_{\text {shear }} \mathbf{u}_{\mathrm{h}}=\mathbf{0}\right\}
$$

where $I_{\mathrm{h}}^{\mathrm{s}}$ is the subspace of possible deformations associated with a null transverse shear strain field, and $U_{\mathrm{h}}$ represents an approximation of the linear space of all admissible solutions $U$.

To avoid the occurrence of transverse shear-locking effects, the discrete finite element solution $\mathbf{u}_{\mathrm{h}} \equiv \mathbf{d}_{\mathrm{e}}$ must lie on the null space of $\mathbf{Q}_{\text {shear }}$. In other words, any required displacement solution must be a linear combination from the components of a given basis of $I_{\mathrm{h}}^{\mathrm{s}}[25,33]$. If this is not the case, the onset of shear locking is verified.

Distinct finite element formulations, for a given mesh, will lead to distinct null transverse shear strain subspaces. In the next sections an analysis over the bilinear degenerated, mixed interpolated MITC4 and bilinear selective reduced elements is performed, aiming to identify for each one of the elements their respective subspace $I_{\mathrm{h}}^{\mathrm{s}}$ and, consequently, to clarify their ability (or not) to avoid locking behaviours.

\subsection{Analysis of the degenerated element}

Referring to Equation (9), it is possible to state for a single element (dropping the elemental indices) that

$$
\left\{\begin{array}{l}
\mathbf{E}_{\xi \zeta} \\
\mathbf{E}_{\eta \zeta}
\end{array}\right\}=\left[\begin{array}{l}
\mathbf{Q}_{\xi \zeta} \\
\mathbf{Q}_{\eta \zeta}
\end{array}\right] \mathbf{d}=\mathbf{0}
$$

where the former matrix $\mathbf{Q}_{\text {shear }}$ was decomposed into two sub-matrices $\mathbf{Q}_{\xi \zeta}$ and $\mathbf{Q}_{\eta \zeta}$.

In the following analysis, a square element placed in the $x y$ plane is considered. The normal to the surface of the element is taken coincident with the $z$-axis, which is also responsible for thickness orientation. The element represents then a reference square in the natural co-ordinate system, with representative dimensions

$$
D_{\text {ref }}=[-1,+1] \times[-1,+1]=\left\{(\xi, \eta) \in \Re^{2} ;-1 \leqslant \xi, \eta \leqslant+1\right\}
$$


With the following configuration, the in-plane $\{u v\}$ components of the displacement vector (2) do not interfere with the transverse shear strain field. It is obvious that any deformation in which only the in-plane degrees of freedom are active will belong to $I_{\mathrm{h}}^{\mathrm{s}}$. Therefore, for the sake of simplicity, these degrees of freedom will be 'removed' from the generalized nodal displacement vector (2), leading to the 'reduced' vector

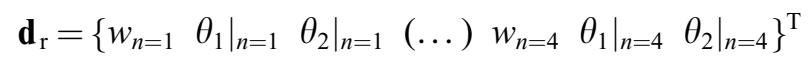

specific to the bilinear element and associated with the condition

$$
\left\{\begin{array}{l}
\mathbf{E}_{\xi \zeta} \\
\mathbf{E}_{\eta \zeta}
\end{array}\right\}=\left[\begin{array}{l}
\mathbf{Q}_{\zeta \zeta} \\
\mathbf{Q}_{\eta \zeta}
\end{array}\right] \mathbf{d}_{\mathrm{r}}=\mathbf{0}
$$

in replacement of (11).

The next step refers to the extension of this equality over all the sample points used in the numerical integration. For the four-node bilinear element, with a complete $2 \times 2$ Gauss integration, 8 restrictions in the form of (14) are introduced and two expressions can be obtained as

$$
\left\{\begin{array}{c}
E_{\xi \zeta}\left(\xi_{\mathrm{I}}, \eta_{\mathrm{I}}\right) \\
E_{\xi \zeta}\left(\xi_{\mathrm{II}}, \eta_{\mathrm{II}}\right) \\
E_{\xi \zeta}\left(\xi_{\mathrm{III}}, \eta_{\mathrm{III}}\right) \\
E_{\xi \zeta}\left(\xi_{\mathrm{IV}}, \eta_{\mathrm{IV}}\right)
\end{array}\right\}=\underbrace{\left[\begin{array}{c}
\mathbf{Q}_{\xi \zeta}\left(\xi_{\mathrm{I}}, \eta_{\mathrm{I}}\right)^{\mathrm{T}} \\
\mathbf{Q}_{\xi \zeta}\left(\xi_{\mathrm{II}}, \eta_{\mathrm{II}}\right)^{\mathrm{T}} \\
\mathbf{Q}_{\xi \zeta}\left(\xi_{\mathrm{III}}, \eta_{\mathrm{III}}\right)^{\mathrm{T}} \\
\mathbf{Q}_{\xi \zeta}\left(\xi_{\mathrm{IV}}, \eta_{\mathrm{IV}}\right)^{\mathrm{T}}
\end{array}\right]}_{4 \times 12} \mathbf{d}_{\mathrm{r}}=\mathbf{0}
$$

and

$$
\left\{\begin{array}{c}
E_{\eta \zeta}\left(\xi_{\mathrm{I}}, \eta_{\mathrm{I}}\right) \\
E_{\eta \zeta}\left(\xi_{\mathrm{II}}, \eta_{\mathrm{II}}\right) \\
E_{\eta \zeta}\left(\xi_{\mathrm{III}}, \eta_{\mathrm{III}}\right) \\
E_{\eta \zeta}\left(\xi_{\mathrm{IV}}, \eta_{\mathrm{IV}}\right)
\end{array}\right\}=\underbrace{\left[\begin{array}{c}
\mathbf{Q}_{\eta \zeta}\left(\xi_{\mathrm{I}}, \eta_{\mathrm{I}}\right)^{\mathrm{T}} \\
\mathbf{Q}_{\eta \zeta}\left(\xi_{\mathrm{II}}, \eta_{\mathrm{II}}\right)^{\mathrm{T}} \\
\mathbf{Q}_{\eta \zeta}\left(\xi_{\mathrm{III}}, \eta_{\mathrm{III}}\right)^{\mathrm{T}} \\
\mathbf{Q}_{\eta \zeta}\left(\xi_{\mathrm{IV}}, \eta_{\mathrm{IV}}\right)^{\mathrm{T}}
\end{array}\right]}_{4 \times 12} \mathbf{d}_{\mathrm{r}}=\mathbf{0}
$$

where each pair $\left(\xi_{k}, \eta_{k}\right)$ represents the natural co-ordinates of a specific integration point $(k=\mathrm{I}, \ldots, \mathrm{IV})$. Also, $\mathbf{Q}_{\xi \zeta}\left(\xi_{k}, \eta_{k}\right)$ and $\mathbf{Q}_{\eta \zeta}\left(\xi_{k}, \eta_{k}\right)$ represent vectors (12 components) referring to the same integration point. It is worth noting that $\mathbf{Q}_{\text {shear }}$ evaluation does not depend on the numerical integration over the thickness and, therefore, the $\zeta$ orientation was not taken into account in the following. Therefore a reduced displacement field, in the sense of (13) and satisfying Equations (15) and (16) (simultaneously avoiding the trivial solution $\mathbf{d}_{\mathrm{r}}=\mathbf{0}$ ) is searched.

After these considerations, the analysis of the degenerated four-node element, characterized by a transverse shear strain field derivable from (3), provided a matrix $\mathbf{Q}_{\text {shear }}$ with the following characteristics:

$$
\operatorname{rank}\left(\mathbf{Q}_{\text {shear }}\right)=8, \quad \text { therefore } \operatorname{nullity}\left(\mathbf{Q}_{\text {shear }}\right)=4
$$



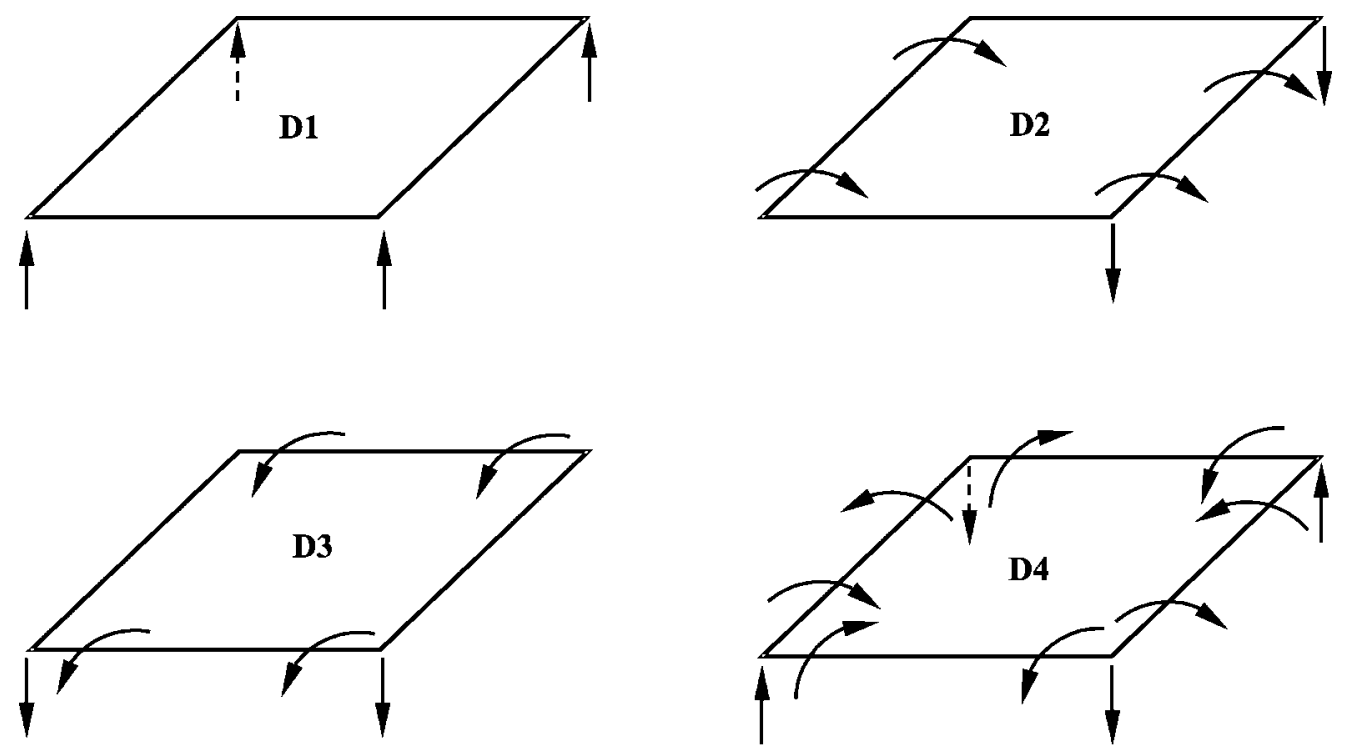

Figure 1. Basis components for the null transverse shear strain subspace; degenerated (D) elements (one element mesh).

As a consequence, the element can reproduce four fundamental displacement patterns. In case a given displacement cannot be obtained as a linear combination of these four terms, transverse shear locking is verified. The displacement configurations forming a basis of the null transverse shear strain subspace for the degenerated element $\left.I_{\mathrm{h}}^{\mathrm{S}}\right|_{\mathrm{D}}$ are schematically represented in Figure 1 . The arrows in the figure refer to activated degrees of freedom. The displacement vector for the whole element (following the representation in Equation (13)) can be represented as follows:

$$
\begin{aligned}
& \mathrm{D} 1 \Rightarrow \mathbf{d}_{\mathrm{r}}=\{1,0,0,1,0,0,1,0,0,1,0,0\}^{\mathrm{T}} \\
& \mathrm{D} 2 \Rightarrow \mathbf{d}_{\mathrm{r}}=\{0,1,0,-1,1,0,-1,1,0,0,1,0\}^{\mathrm{T}} \\
& \mathrm{D} 3 \Rightarrow \mathbf{d}_{\mathrm{r}}=\{-1,0,1,-1,0,1,0,0,1,0,0,1\}^{\mathrm{T}} \\
& \mathrm{D} 4 \Rightarrow \mathbf{d}_{\mathrm{r}}=\{1,2,-2,-1,2,2,1,-2,2,-1,-2,-2\}^{\mathrm{T}}
\end{aligned}
$$

The element is able, then, to reproduce these displacement patterns and also any linear combinations between them.

\subsection{Analysis of the mixed interpolated bilinear element}

The described approach is now applied into the four-node bilinear element of Dvorkin and Bathe [10]. The concept behind the element formulation is well established in the finite element literature (see, for instance, References [2, 10, 12, 14, 31] for details) being only summarized here. 
The assumed natural strain approach (or, as originally stated, mixed interpolation of tensorial components) applied to the four-node element consists in the reduction of the bilinear transverse shear fields to a constant linear one, based on sampling points for each of the two shear strain components [5]. In a general sense, the interpolated transverse shear strains can be defined as

$$
\begin{aligned}
& \mathbf{E}_{\xi \zeta}^{\mathrm{ANS}}(\xi, \eta, \zeta)=\sum_{p=1}^{2} N_{p}(\xi, \eta) \mathbf{E}_{\xi \zeta}^{\mathrm{u}}\left(\xi_{p}, \eta_{p}, 0\right) \\
& \mathbf{E}_{\eta \zeta}^{\mathrm{ANS}}(\xi, \eta, \zeta)=\sum_{q=1}^{2} N_{q}(\xi, \eta) \mathbf{E}_{\eta \zeta}^{\mathrm{u}}\left(\xi_{q}, \eta_{q}, 0\right)
\end{aligned}
$$

function of the displacement evaluated strains of Equation (3). In the latter equations, $(p)$ and $(q)$ represent additional sampling points with natural co-ordinates $\left(\xi_{p}, \eta_{p}\right)$ and $\left(\xi_{q}, \eta_{q}\right)$, along with their respective shape functions $\left(N_{p}\right)$ and $\left(N_{q}\right)$.

The analysis of Equations (15) and (16) for this element reveals a matrix $\mathbf{Q}_{\text {shear }}$ with the following characteristics:

$$
\operatorname{rank}\left(\mathbf{Q}_{\text {shear }}\right)=4, \quad \text { therefore } \operatorname{nullity}\left(\mathbf{Q}_{\text {shear }}\right)=8
$$

The null transverse shear strain subspace $\left.I_{\mathrm{h}}^{\mathrm{s}}\right|_{M}$ for the mixed interpolated MITC4 element should, then, be represented by a basis with 8 terms, being a possible candidate represented in Figure 2, with the respective displacement vectors as follows:

$$
\begin{aligned}
& \mathrm{M} 1 \Rightarrow \mathbf{d}_{\mathrm{r}}=\{1,0,0,1,0,0,1,0,0,1,0,0\}^{\mathrm{T}} \\
& \mathrm{M} 2 \Rightarrow \mathbf{d}_{\mathrm{r}}=\{0,1,0,-1,1,0,-1,1,0,0,1,0\}^{\mathrm{T}} \\
& \mathrm{M} 3 \Rightarrow \mathbf{d}_{\mathrm{r}}=\{-1,0,1,-1,0,1,0,0,1,0,0,1\}^{\mathrm{T}} \\
& \mathrm{M} 4 \Rightarrow \mathbf{d}_{\mathrm{r}}=\{1,2,-2,-1,2,2,1,-2,2,-1,-2,-2\}^{\mathrm{T}} \\
& \mathrm{M} 5 \Rightarrow \mathbf{d}_{\mathrm{r}}=\{0,1,0,0,-1,0,0,0,0,0,0,0\}^{\mathrm{T}} \\
& \mathrm{M} 6 \Rightarrow \mathbf{d}_{\mathrm{r}}=\{0,0,0,0,0,0,0,-1,0,0,1,0\}^{\mathrm{T}} \\
& \mathrm{M} 7 \Rightarrow \mathbf{d}_{\mathrm{r}}=\{0,0,-1,0,0,0,0,0,0,0,0,1\}^{\mathrm{T}} \\
& \mathrm{M} 8 \Rightarrow \mathbf{d}_{\mathrm{r}}=\{0,0,0,0,0,-1,0,0,1,0,0,0\}^{\mathrm{T}}
\end{aligned}
$$

It can be noted that the first four terms of MITC4 subspace basis are exactly the same of the degenerated element. The remaining four terms do not belong to the null transverse shear strain subspace of the degenerated element.

\subsection{The selective reduced element}

Starting with the degenerated element, an additional analysis is performed over the selective reduced integration procedure [6,7]. For this purpose, a reduced integration (one point quadrature) is adopted for the calculation of the transverse shear strain terms. 

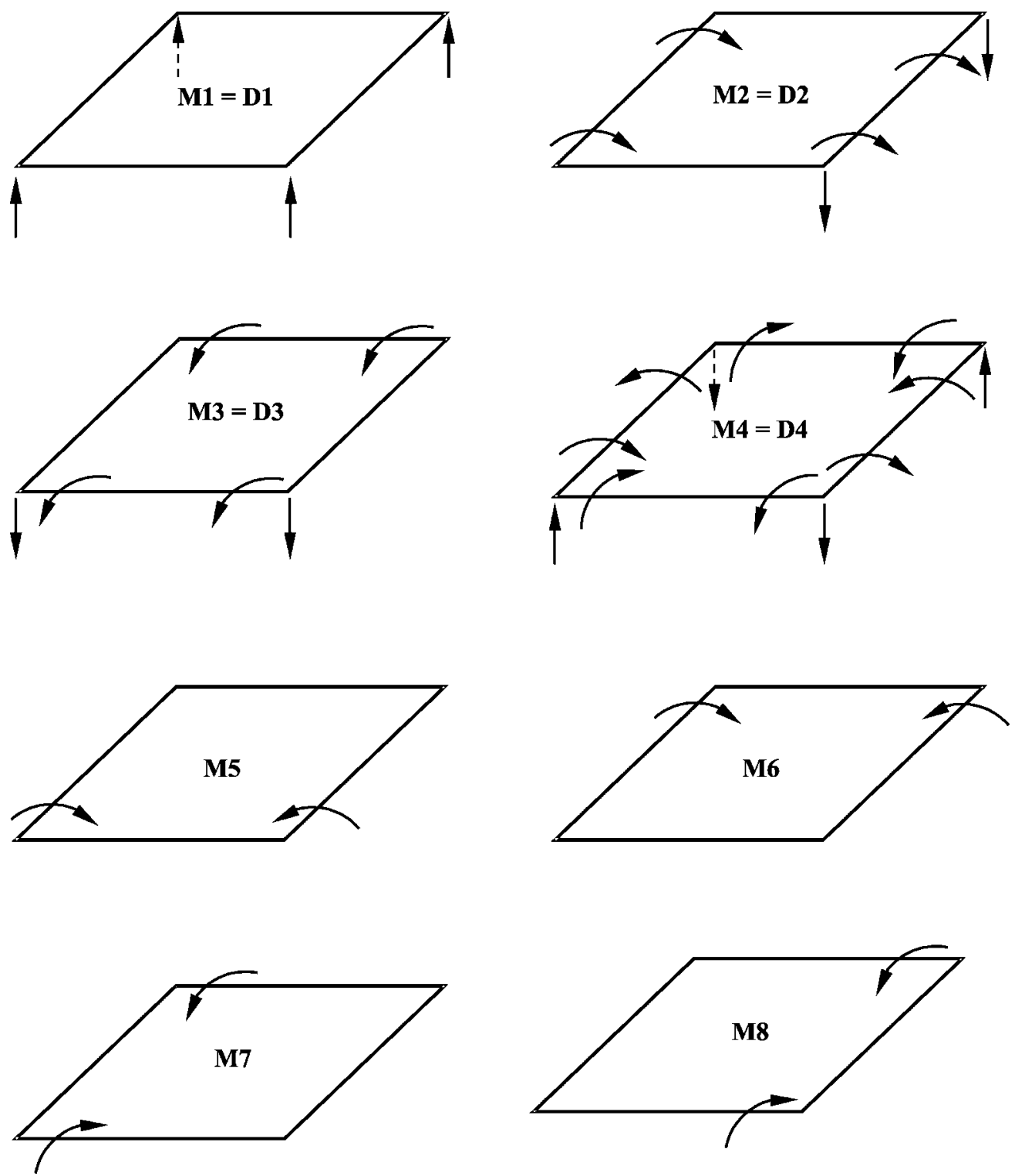

Figure 2. Basis components for the null transverse shear strain subspace; MITC4 (M) elements (one element mesh).

The analysis of this formulation with Equations (15) and (16) leads to a matrix $\mathbf{Q}_{\text {shear }}$ characterized by

$$
\operatorname{rank}\left(\mathbf{Q}_{\text {shear }}\right)=2, \quad \text { therefore } \operatorname{nullity}\left(\mathbf{Q}_{\text {shear }}\right)=10
$$



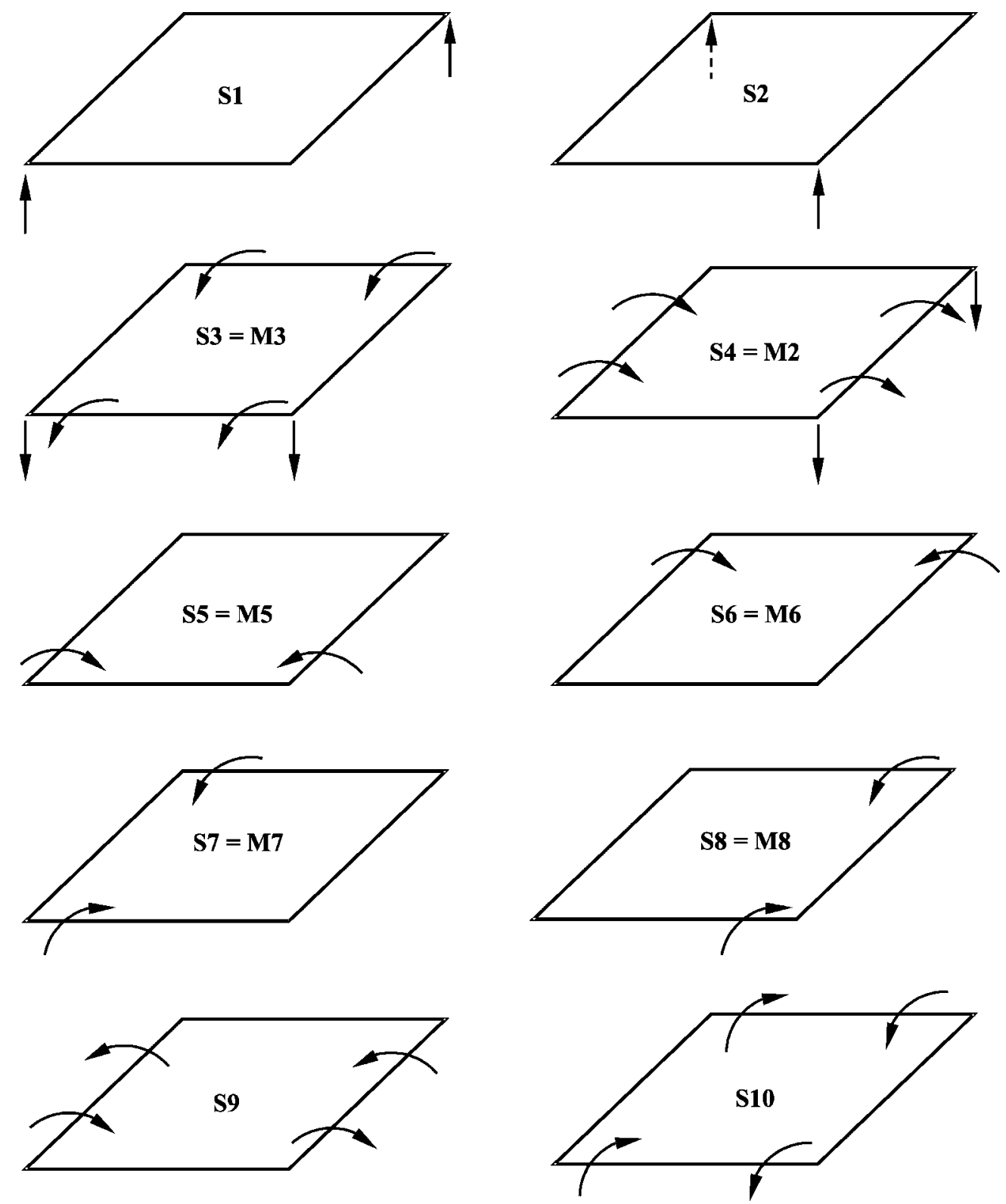

Figure 3. Basis components for the null transverse shear strain subspace; selectively reduced integrated (S) elements (one element mesh).

A possible basis, defining the null shear strain subspace $\left.I_{\mathrm{h}}^{\mathrm{s}}\right|_{\mathrm{S}}$ for the $S R I$ element, is reproduced in Figure 3, with the corresponding displacement configurations

$$
\mathrm{S} 1 \Rightarrow \mathbf{d}_{\mathrm{r}}=\{1,0,0,0,0,0,1,0,0,0,0,0\}^{\mathrm{T}}
$$




$$
\begin{aligned}
\mathrm{S} 2 & \Rightarrow \mathbf{d}_{\mathrm{r}}=\{0,0,0,1,0,0,0,0,0,1,0,0\}^{\mathrm{T}} \\
\mathrm{S} 3 & \Rightarrow \mathbf{d}_{\mathrm{r}}=\{-1,0,1,-1,0,1,0,0,1,0,0,1\}^{\mathrm{T}} \\
\mathrm{S} 4 & \Rightarrow \mathbf{d}_{\mathrm{r}}=\{0,1,0,-1,1,0,-1,1,0,0,1,0\}^{\mathrm{T}} \\
\mathrm{S} 5 & \Rightarrow \mathbf{d}_{\mathrm{r}}=\{0,1,0,0,-1,0,0,0,0,0,0,0\}^{\mathrm{T}} \\
\mathrm{S} 6 & \Rightarrow \mathbf{d}_{\mathrm{r}}=\{0,0,0,0,0,0,0,-1,0,0,1,0\}^{\mathrm{T}} \\
\mathrm{S} 7 & \Rightarrow \mathbf{d}_{\mathrm{r}}=\{0,0,-1,0,0,0,0,0,0,0,0,1\}^{\mathrm{T}} \\
\mathrm{S} 8 & \Rightarrow \mathbf{d}_{\mathrm{r}}=\{0,0,0,0,0,-1,0,0,1,0,0,0\}^{\mathrm{T}} \\
\mathrm{S} 9 & \Rightarrow \mathbf{d}_{\mathrm{r}}=\{0,1,0,0,1,0,0,-1,0,0,-1,0\}^{\mathrm{T}} \\
\mathrm{S} 10 & \Rightarrow \mathbf{d}_{\mathrm{r}}=\{0,0,-1,0,0,1,0,0,1,0,0,-1\}^{\mathrm{T}}
\end{aligned}
$$

The subspace basis obtained with this formulation is composed of 10 terms. As described in the figure, terms (S3)-(S8) are included in the subspace basis of the MITC4 element. Additionally, terms M1 and M4 of this last element can be obtained as a linear combination of the $(\mathrm{S})$ terms above in the form

$$
\mathrm{M} 1=\mathrm{S} 1+\mathrm{S} 2
$$

and

$$
\mathrm{M} 4=\alpha_{1} \mathrm{~S} 1+\alpha_{2} \mathrm{~S} 2+\alpha_{3} \mathrm{~S} 9+\alpha_{4} \mathrm{~S} 10, \quad\left(\alpha_{1}, \alpha_{2}, \alpha_{3}, \alpha_{4} \in \Re^{3}\right)
$$

From all the previous analysis, it can be verified that the subspace for the SRI formulation encompasses the previous ones as the statement

$$
\left.\left.\left.I_{\mathrm{h}}^{\mathrm{s}}\right|_{\mathrm{S}} \supset I_{\mathrm{h}}^{\mathrm{s}}\right|_{\mathrm{M}} \supset I_{\mathrm{h}}^{\mathrm{s}}\right|_{\mathrm{D}}
$$

is verified.

In addition, from Figure 3 it is interesting to note that some deformation patterns pertaining to selective reduced integration element are not included amongst the MITC4 and degenerated element bases, and cannot be spanned by either of them.

For these patterns, the mixed interpolation element will verify the occurrence of locking phenomenon, performing in the very same manner as the degenerated element. Based on this conclusion, two example problems are proposed. The adopted values for the examples are merely indicative and therefore only a qualitative solution is sought, with no physical meaning intended.

2.4.1. Example 2.1. Consider a single bilinear square element with sides measuring two units $(L=2)$, so that no distinction needs to be made between physical and isoparametric co-ordinates. The element possesses a thickness value of $(h=0.0002)$ and the material properties adopted are a Young's modulus $\left(E=2.11 \times 10^{11}\right)$ and a Poisson coefficient $(v=0.3)$.

The element is restrained in such a way that only one type of rotations per node (rotation type $\theta_{1}$, as defined in Equation (13)) is allowed as shown in Figure 4. In the figure the specific rotations activated amongst the total degrees of freedom are indicated. The arrows also correspond to applied unity moments.

Comparing the last figure with the patterns represented in Figures 1, 2 and 3, it is possible to retain some conclusions. First of all the degenerated element cannot reproduce the imposed deformation from any linear combination of the patterns represented in Figure 1. Relating to 


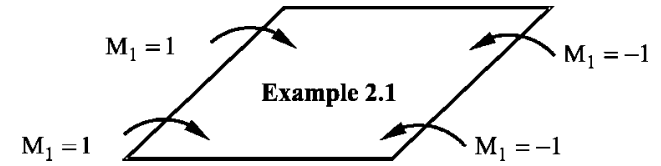

Figure 4. Allowed degrees of freedom for Example 2.1; arrows also indicate directions of the applied unity moments.

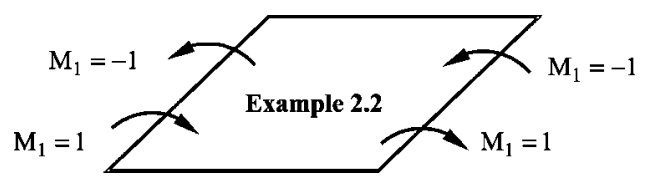

Figure 5. Allowed degrees of freedom for Example 2.2; arrows also indicate directions of the applied unity moments.

the mixed interpolation and selective reduced integration formulations this does not happen, with the deformation pattern being easily obtained in the form

$$
\text { Example } 2.1 \rightarrow\left\{\begin{array}{l}
\mathrm{M} 5+\mathrm{M} 6 \\
\mathrm{~S} 5+\mathrm{S} 6
\end{array}\right.
$$

Doing so, both MITC4 and SRI elements are supposed to reproduce the configuration imposed, while the degenerated element is expected to lock. In fact, applying unit moments over the rotational degrees of freedom depicted in the figure the following results for the corresponding rotations are obtained:

$$
\begin{aligned}
\text { Degenerated element } & \rightarrow \text { rotation }\left|\theta_{1}\right|=2.21801 \times 10^{-7} \\
\text { MITC4 element } & \rightarrow \text { rotation }\left|\theta_{1}\right|=6.46919 \\
\text { SRI element } & \rightarrow \text { rotation }\left|\theta_{1}\right|=6.46919
\end{aligned}
$$

Qualitatively, the discrepancy is patent between the values obtained for the degenerated and MITC4/SRI elements. These latter elements present the same response to the imposed load and boundary conditions, as expected from the null transverse shear strain subspace analysis.

2.4.2. Example 2.2. The same geometry, material and boundary conditions from the previous example are now applied in this test. The only difference is the orientation of the applied unity-value moments, now represented in Figure 5.

It can be seen that the analysed configuration can only be obtained from the SRI formulation (term S9 in Figure 3). Once neither the degenerated nor the mixed interpolated elements can reproduce the present orientation, it is supposed that both elements reveal a tendency to lock. In fact this is what actually happens, as can be verified with the following results:

$$
\begin{aligned}
\text { Degenerated element } & \rightarrow \text { rotation }\left|\theta_{1}\right|=2.21801 \times 10^{-7} \\
\text { MITC4 element } & \rightarrow \text { rotation }\left|\theta_{1}\right|=2.21801 \times 10^{-7} \\
\text { SRI element } & \rightarrow \text { rotation }\left|\theta_{1}\right|=18.48340
\end{aligned}
$$

The obtained values reveal a poor behaviour of the assumed natural strain formulation, in opposition of the previous example, with MITC4 and the degenerated element leading to the same numerical results. 

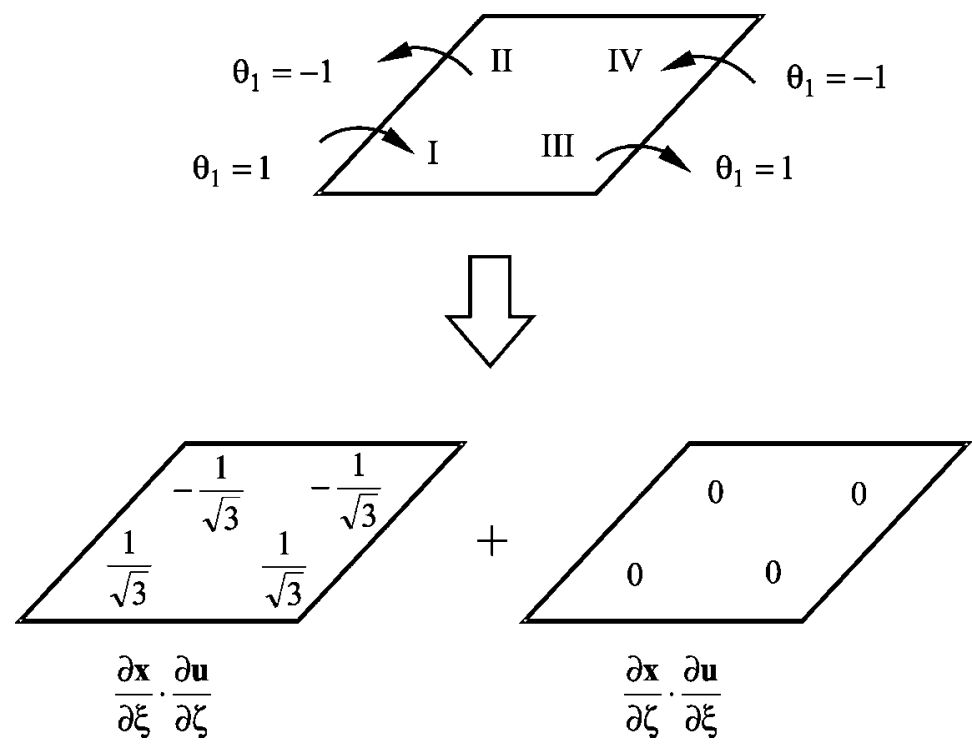

Figure 6. Shear strain $E_{\xi \zeta}^{\mathrm{u}}$ at Gauss points (I, II, III, IV) for the degenerated shell element.

About the SRI element, as its null shear strain subspace reproduces the proposed deformation, no shear locking effects are verified.

\section{TREATMENT OF THE TRANSVERSE SHEAR LOCKING USING THE EAS METHOD}

Following a previous work in 2D analysis [25], an enhanced approach acting on the transverse shear strains is adopted by the use of compatible displacement fields, adopting the well-known bubble function

$$
N_{\alpha}(\xi, \eta)=\left(1-\xi^{2}\right)\left(1-\eta^{2}\right)
$$

In order to exemplify the following procedures, component (S9) of the null shear strain subspace for the SRI formulation (described in (24) and in Example 2.2) is once again analysed. However, instead of moments, unity values rotations are applied over the activated degrees of freedom (Figure 6). Focusing on the $E_{\xi \zeta}^{\mathrm{u}}$ transverse shear strain term, from Equations (3) and (4) it can be stated that

$$
2 E_{\xi \zeta}^{\mathrm{u}}=\left(\frac{\partial \mathbf{x}}{\partial \xi} \cdot \frac{\partial \mathbf{u}}{\partial \zeta}\right)+\left(\frac{\partial \mathbf{x}}{\partial \zeta} \cdot \frac{\partial \mathbf{u}}{\partial \xi}\right)
$$

Adopting the same geometric and material configuration of Example 2.2, the displacement based shear strain in Equation (26) is obtained for each Gauss point for the degenerated element. From the subspace analysis described in the previous sections, it is clear that this element cannot reproduce the imposed displacement pattern. 
The deformation values obtained can then be split over the contributions of each term on the right-hand side of Equation (26), with a schematic representation presented in Figure 6. As it can be seen, shear strain values are not null at Gauss points for this boundary-load configuration, as it would be expected from the subspace basis evaluated at point 2.2.

In order to be possible to force the element to obey the Kirchhoff condition (7) an enhanced strain field of the form

$$
\overline{\mathbf{E}}(\xi)=\mathbf{M}(\xi) \boldsymbol{\alpha}_{\mathrm{e}}
$$

can be added up, with the resulting strain being defined, in the isoparametric space, as

$$
E_{\xi \zeta}=E_{\xi \zeta}^{\mathrm{u}}+\bar{E}_{\xi \zeta}
$$

In Equation (27), $\mathbf{M}(\xi)$ defines the interpolation matrix relating the enhanced strain field and the vector of internal variables $\alpha_{\mathrm{e}}$ in the isoparametric space, as in the work of Simo and Rifai [16]. The enhanced strain in physical space can then be obtained with a similar discrete relation in the form

$$
\bar{\varepsilon}_{\mathrm{e}}=\mathbf{M} \boldsymbol{\alpha}_{\mathrm{e}}
$$

with the M matrix defined by transformation rules for rank two tensors (for details, see References $[5,16])$.

With this procedure Equation (28) now provides the missing term for expression (26), forcing the summation represented in Figure 6 to be zero. The same reasoning is also set for the $E_{\eta \zeta}^{\mathrm{u}}$ analysis, which will include (S10) term into $\left.I_{\mathrm{h}}^{\mathrm{s}}\right|_{\mathrm{D}}$ for the degenerated element.

Finally, an optimized expression can be developed for the transverse enhanced shear strain field in the form

$$
\left\{\begin{array}{l}
\bar{E}_{\xi \zeta} \\
\bar{E}_{\eta \zeta}
\end{array}\right\}_{2}=\left.\left.\mathbf{M}(\xi, \eta)\right|_{2} \quad \boldsymbol{\alpha}\right|_{2}=\left[\begin{array}{cc}
\frac{\partial N_{\alpha}}{\partial \eta} & 0 \\
0 & \frac{\partial N_{\alpha}}{\partial \xi}
\end{array}\right]\left\{\begin{array}{l}
\alpha_{1} \\
\alpha_{2}
\end{array}\right\}
$$

based on the partial $(\xi, \eta)$ derivatives of the bubble function (25) and on the use of a twocomponent internal variable vector $\left(\left.\boldsymbol{\alpha}\right|_{2}\right)$.

The enhancement vector (30) is used, then, to define the total natural strain field, through a generalization of expression (28) in the form

$$
\left\{\begin{array}{c}
E_{\xi \zeta} \\
E_{\eta \zeta}
\end{array}\right\}=\left\{\begin{array}{l}
E_{\xi \zeta}^{u} \\
E_{\eta \zeta}^{u}
\end{array}\right\}+\left\{\begin{array}{l}
\bar{E}_{\xi \zeta} \\
\bar{E}_{\eta \zeta}
\end{array}\right\}_{2}
$$

With the preceding enriched expression, the subspace dimension for the degenerated element becomes enlarged, now embracing (S9) and (S10) basis terms, originally belonging only to the SRI formulation.

\subsection{The S4E4 and S4E4_P7 shell elements}

The four-node degenerated element, after the enhancement analysis performed in the previous section, presents an increased subspace dimension. However, an important part of deformation 
configurations is still missing when compared to MITC4 and SRI formulations. These configurations refer to components (S5), (S6), (S7) and (S8) of the subspace basis represented in Figure 3.

With the EAS method, and following the previous developments, it is possible to conceive a total shear strain field that simultaneously encompasses these four terms and also the two patterns treated before. To accomplish this objective, a new enhanced shear strain field is introduced in the form

$$
\left\{\begin{array}{c}
\bar{E}_{\xi \zeta} \\
\bar{E}_{\eta \zeta}
\end{array}\right\}_{4}=\left.\left.\mathbf{M}(\xi, \eta)\right|_{4} \quad \boldsymbol{\alpha}\right|_{4}=\left[\begin{array}{cccc}
\frac{\partial N_{\alpha}}{\partial \eta} & 0 & \frac{\partial N_{\alpha}}{\partial \xi} & 0 \\
0 & \frac{\partial N_{\alpha}}{\partial \xi} & 0 & \frac{\partial N_{\alpha}}{\partial \eta}
\end{array}\right]\left\{\begin{array}{l}
\alpha_{1} \\
\alpha_{2} \\
\alpha_{3} \\
\alpha_{4}
\end{array}\right\}
$$

As can be immediately noticed, the $\left.\mathbf{M}(\xi, \eta)\right|_{4}$ matrix above comprises the $\left.\mathbf{M}(\xi, \eta)\right|_{2}$ stated in Equation (30). This four-variable enhancement, with the correspondent internal variables field $\left(\left.\boldsymbol{\alpha}\right|_{4}\right)$, now allows the 6 deformation configurations that were previously missing ((S5), (S6), (S7) and (S8) now included and the previous analysed (S9) and (S10)), with the total strain field obtained by generalization of Equation (31).

The explanation why this four-variable enhancement provides six additional displacement patterns for the basis of the null transverse shear strain subspace comes from the fact that the eight restrictions (as defined in (15) and (16)), imposing the null shear strain at the Gauss points, result now in only six linear independent equations. As the effective number of elemental degrees of freedom is 16 (12 from the reduced vector (13) plus 4 internal variables), the final number of displacement configurations turns out to be 10 .

At this point, it is possible to introduce two new four-node bilinear shell elements, both of them based on the original degenerated formulation. The first element possesses an enhancement in the form (32) and is termed S4E4, due to the number of nodes and internal variables, the $(E)$ letter referring to shear strain enhancement only. The second element proposed encompasses not only the preceding shear strain enrichment but also the in-plane enhancement introduced by Andelfinger and Ramm [5]. In the following examples, this element is labelled S4E4_P7, where the previous notation was extending to accommodate their in-plane approach (P letter) using seven internal variables. Following Reference [5] the interpolation matrix for the in-plane enhancement is defined as

$$
\mathbf{M}(\xi) \equiv \mathbf{M}(\xi, \eta)=\left[\begin{array}{ccccccc}
\xi & 0 & 0 & 0 & \xi \eta & 0 & 0 \\
0 & \eta & 0 & 0 & 0 & \xi \eta & 0 \\
0 & 0 & \xi & \eta & 0 & 0 & \xi \eta
\end{array}\right]
$$

\subsection{The S4E6 and S4E6_P7 shell elements}

The developed subspace analysis guided so far the choice of the enhanced terms to be included in the transverse shear strain field. As can be stated from the examples presented at the end of this work, the obtained elements with four additional internal variables provided an improvement over the original degenerated element. However, their performances were still far from the desired results. 
Using the enhancement terms obtained from the subspace analysis, it was verified that an improved performance can be obtained including in (32) the cross derivatives of the bubble function in order to $\xi$ and $\eta$, leading to a new interpolation matrix of the form

$$
\left.\mathbf{M}(\xi, \eta)\right|_{6}=\left[\begin{array}{cccccc}
\frac{\partial N_{\alpha}}{\partial \eta} & 0 & \frac{\partial N_{\alpha}}{\partial \xi} & 0 & \frac{\partial N_{\alpha}}{\partial \xi} \frac{\partial N_{\alpha}}{\partial \eta} & 0 \\
0 & \frac{\partial N_{\alpha}}{\partial \xi} & 0 & \frac{\partial N_{\alpha}}{\partial \eta} & 0 & \frac{\partial N_{\alpha}}{\partial \xi} \frac{\partial N_{\alpha}}{\partial \eta}
\end{array}\right]
$$

The matrix $\left.\mathbf{M}(\xi, \eta)\right|_{6}$ can then be related, within the context of the present work, to an optimal choice for the enhanced interpolation, with the internal variable field now being represented as a vector with six components.

Similarly to before, the elements obtained from this formulation can be termed S4E6 and S4E6_P7, as only shear or shear plus in-plane enhancement is present, respectively.

\subsection{A different approach: the MITC4_E2 shell element}

Changing the approach adopted herein, the MITC4 element of Dvorkin and Bathe [10] is now focused. As detailed in the analysis of the null transverse shear strain subspace described in Section 2.3, the chosen basis of the element has two missing components when compared to SRI formulation, namely the (S9) and (S10) terms of Figure 3. As detailed in the beginning of this section, these two components can be included into the deformation field with an enhanced process using two internal variables (Equation (30)). In this way, it is possible to improve the performance of the MITC4 shell element via the EAS method over the transverse shear strains. The shell finite element so obtained is termed MITC4_E2, following the previous notation, and possesses a total transverse strain field in the form

$$
\left\{\begin{array}{c}
E_{\xi \zeta} \\
E_{\eta \zeta}
\end{array}\right\}=\left\{\begin{array}{l}
E_{\xi \zeta}^{\mathrm{ANS}} \\
E_{\eta \zeta}^{\mathrm{ANS}}
\end{array}\right\}+\left\{\begin{array}{c}
\bar{E}_{\xi \zeta} \\
\bar{E}_{\eta \zeta}
\end{array}\right\}
$$

with the assumed natural strain terms being as in Equations (19) and (20) and the interpolation matrix used in the enhanced strain field defined in Equation (30). As it would be expected, a new analysis of Example 2.2 using this element provides the same result as the SRI formulation.

\section{EXAMPLES FOR ELEMENTS ASSESSMENT}

In order to assess the performance of the proposed shell elements, several problems selected from the literature are analysed. The obtained results are compared to those previously published using well-known shell element formulations. A list of the analysed shell elements and the abbreviations used to identify them henceforth is contained in Table I.

\subsection{Morley's $30^{\circ}$ Skew plate}

The skew, simply supported plate originally proposed by Morley [34] is analysed in order to test the sensitivity of the proposed elements to mesh distortions. The geometric, material and load data $\left(L=100, h=1, E=10^{5}, v=0.3\right.$, uniform pressure load $\left.Q=1\right)$ are based on the work of Andelfinger and Ramm [5], being represented in Figure 7. 
Table I. List of referenced and proposed shell elements.

\begin{tabular}{|c|c|}
\hline Name & Description \\
\hline AHMA4 & Bilinear degenerated shell element, based on the approach of Ahmad et al. [1] \\
\hline EAS7_ANS & In-plane bilinear enhanced shell element; Andelfinger and Ramm [5] \\
\hline MITC4 & Mixed interpolated bilinear shell element; Dvorkin and Bathe [10] \\
\hline SIMO_1990 & Enhanced shear formulation, bilinear element; Simo et al. [16] \\
\hline \multicolumn{2}{|c|}{ Present formulations } \\
\hline S4E4 & $\begin{array}{l}\text { Bilinear degenerated shell element; enhanced transverse shear formulation with } 4 \text { internal } \\
\text { variables }\end{array}$ \\
\hline S4E4_P7 & $\begin{array}{l}\text { Bilinear degenerated shell element; enhanced transverse shear formulation with } 4 \text { internal } \\
\text { variables and enhanced in-plane formulation of Andelfinger and Ramm [5] }\end{array}$ \\
\hline S4E6 & $\begin{array}{l}\text { Bilinear degenerated shell element; enhanced transverse shear formulation with } 6 \text { internal } \\
\text { variables }\end{array}$ \\
\hline S4E6_P7 & $\begin{array}{l}\text { Bilinear degenerated shell element; enhanced transverse shear formulation with } 6 \text { internal } \\
\text { variables and enhanced in-plane formulation of Andelfinger and Ramm [5] }\end{array}$ \\
\hline MITC4_E2 & $\begin{array}{l}\text { Original mixed interpolated element from Dvorkin and Bathe [10]; enhanced transverse shear } \\
\text { formulation with } 2 \text { internal variables }\end{array}$ \\
\hline
\end{tabular}

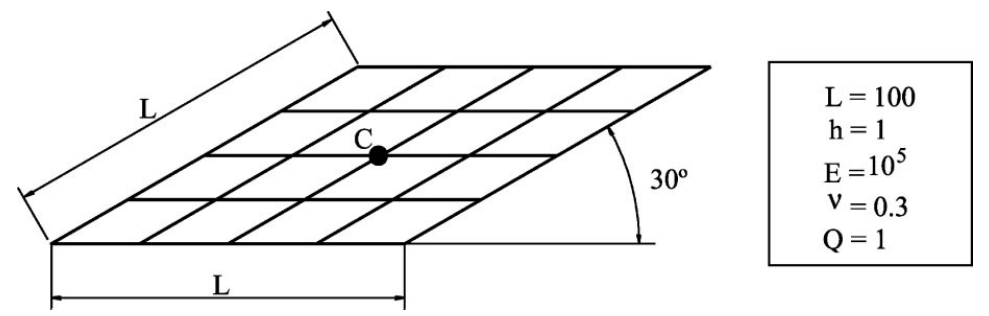

Figure 7. Morley's skew plate.

Still following this work, the Kirchhoff reference solution of 4.455 presented by Morley is replaced by the value 4.640 , as even for the length to thickness ratio of 100 shear deformation effects cannot be neglected.

The present results are compared with the ones obtained by Andelfinger and Ramm (for their EAS7_ANS and Dvorkin and Bathe MITC4 elements). Additionally, the results obtained with the enhanced plate element proposed by Simo and Rifai [16], as implemented by Yunhua and Eriksson in a recent work [20] are showed. The values for the central point deflection of the plate are represented in Table II and graphically in Figure 8. For the sake of completeness, the results obtained with the four-node degenerated shell element are reproduced.

The elements with 6 enhanced variables (S4E6 and S4E6_P7) and also the MITC4_E2 element show remarkably good results even for coarse meshes, leading to the same numerical values. The elements with 4 enhanced variables (S4E4 and S4E4_P7) reveal some sensitivity to coarse meshes, converging to the performance of the other elements as the mesh is refined. 
Table II. Central point (C) deflection of Morley's skew plate.

\begin{tabular}{lccccc}
\hline Mesh & MITC4 & EAS7_ANS & SIMO_1990 & AHMA4 & Ref. solution \\
\hline $4 \times 4$ & 3.9182 & 4.2122 & 3.9305 & 0.1151 & 4.6400 \\
$8 \times 8$ & 3.8991 & 4.2239 & 3.9841 & 0.3841 & 4.6400 \\
$16 \times 16$ & 4.1875 & 4.3738 & 4.2727 & 0.9882 & 4.6400 \\
$32 \times 32$ & 4.4098 & 4.4827 & 4.4668 & 1.9110 & 4.6400 \\
\hline Mesh & S4E4 & S4E4_P7 & S4E6 & S4E6_P7 & MITC4_E2 \\
\hline $4 \times 4$ & 1.9220 & 1.9220 & 4.5090 & 4.5090 & 4.5090 \\
$8 \times 8$ & 3.4530 & 3.4530 & 4.4380 & 4.4380 & 4.4380 \\
$16 \times 16$ & 4.2600 & 4.2600 & 4.4820 & 4.4820 & 4.4820 \\
$32 \times 32$ & 4.5310 & 4.5310 & 4.5610 & 4.5610 & 4.5610 \\
\hline
\end{tabular}

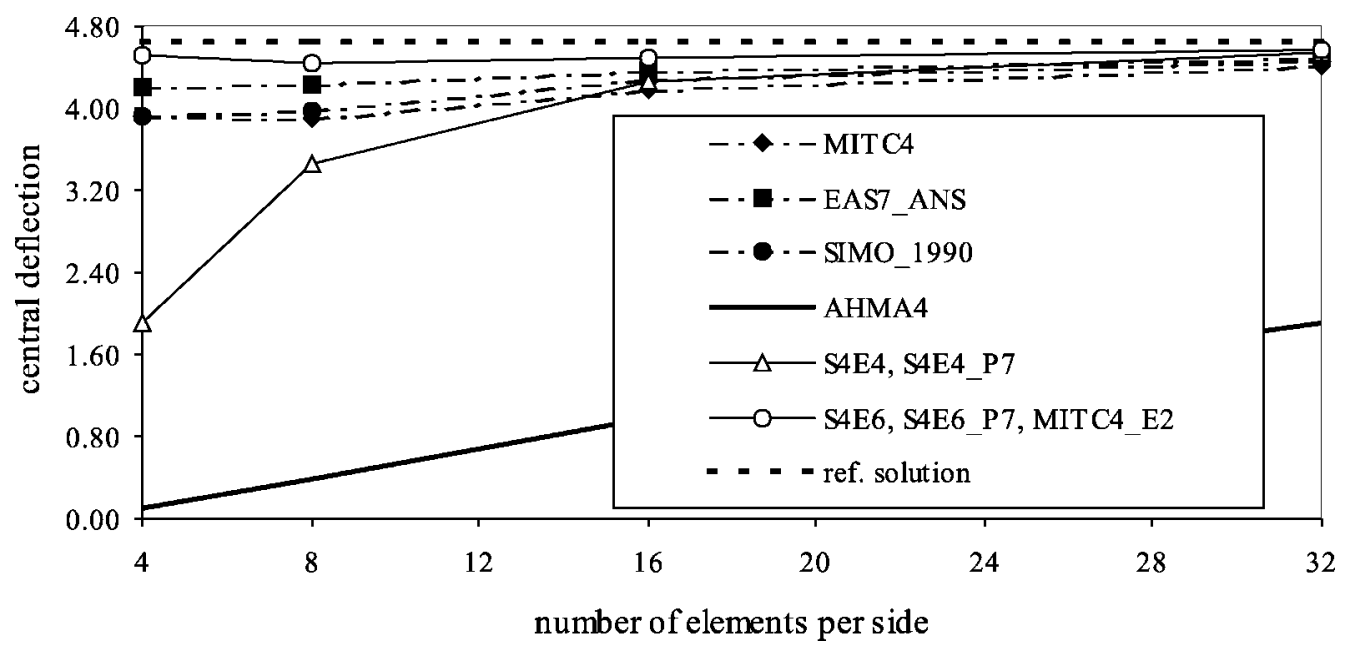

Figure 8. Central point deflection for Morley's skew plate.

\subsection{Scordelis-Lo roof problem}

The geometry of this cylindrical shell, according to Reference [5], is represented in Figure 9, along with the relevant material properties. This data set is also dealt with in Reference [35], reporting to the original work of Scordelis and Lo [36].

The physical basis of the problem is a deeply arched roof supported only by diaphragms at its curved edges (as an aircraft hanger), deforming under its own weight. The geometry is such that the centre point of the roof moves upwards under the self-weight (directed downward) load.

For this problem the vertical displacement at the midpoint of the free edge (point $\mathrm{A}$ in the figure) is taken into account, and a converged numerical solution of 0.3024 is adopted for comparison purposes [35]. Due to symmetry, only one quarter of the structure is considered (dashed zone in the figure). 


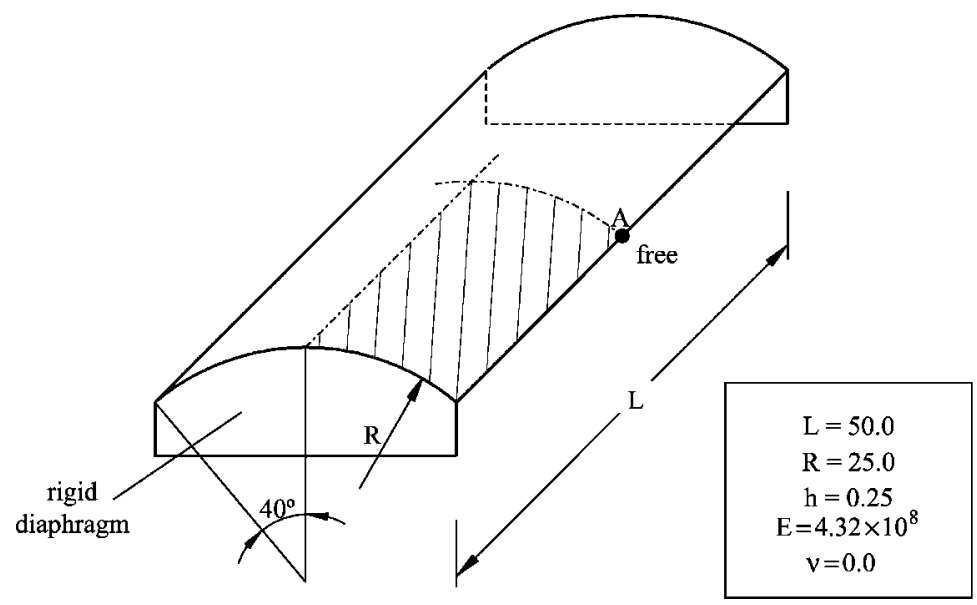

Figure 9. Scordelis-Lo roof.

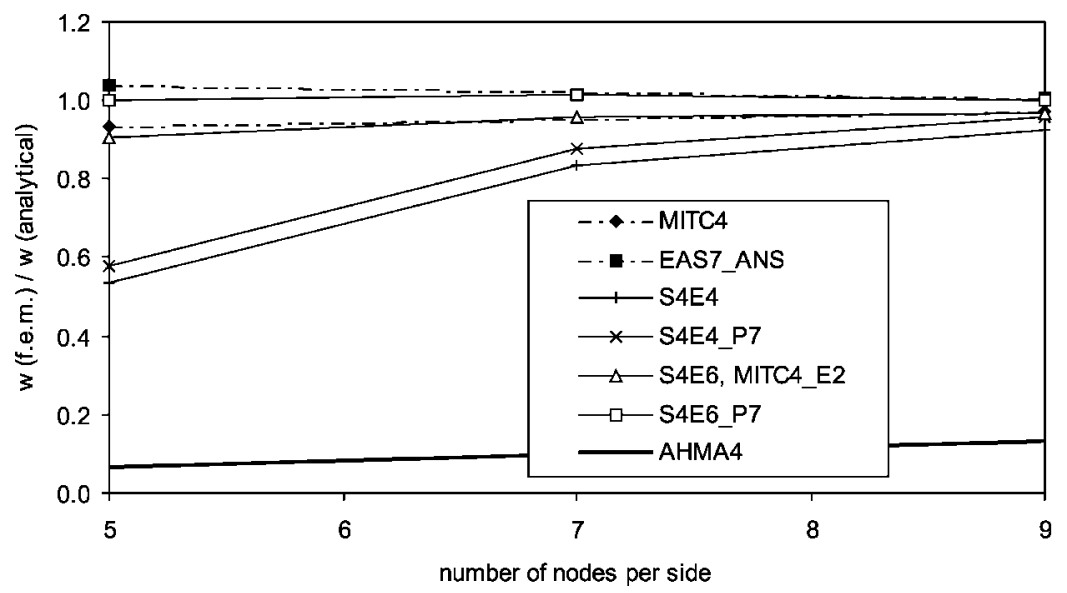

Figure 10. Scordelis-Lo roof. Normalized displacement value at midpoint of free edge (point A).

With these considerations, the obtained results are presented graphically in Figure 10, with the normalized values (ratio between finite elements and analytical solutions) listed in Table III. The results of the presented elements are plotted against the MITC4 element and also in comparison with the EAS7_ANS element.

From the figure and table it is evident the better performance of S4E6_P7 element which, even for the coarser mesh, leads virtually to the correct result. Comparing with EAS7_ANS element, it is patent the improvement obtained with the shear enhancement of the present work in comparison with the assumed natural strain method. Once again it can be verified the good performance of the other enhanced elements proposed, with S4E6 and MITC4_E2 results being almost as good as those obtained with Dvorkin and Bathe element. 
Table III. Scordelis-Lo roof.

\begin{tabular}{lccc}
\hline & \multicolumn{3}{c}{ w(f.e.m.)/w(analytical) -midpoint of free edge } \\
\cline { 2 - 4 } Elements & 5 nodes per side & 7 nodes per side & 9 nodes per side \\
\hline MITC4 & 0.93651 & $*$ & 0.97315 \\
EAS7_ANS & 1.04068 & $*$ & 1.00625 \\
AHMA4 & 0.06806 & 0.10152 & 0.13439 \\
S4E4 & 0.53472 & 0.83565 & 0.92593 \\
S4E4_P7 & 0.57771 & 0.87864 & 0.95602 \\
S4E6 & 0.90542 & 0.95866 & 0.96925 \\
S4E6_P7 & 1.00099 & 1.01323 & 1.00165 \\
MITC4_E2 & 0.90046 & 0.95800 & 0.96892 \\
\hline
\end{tabular}

*Not available.

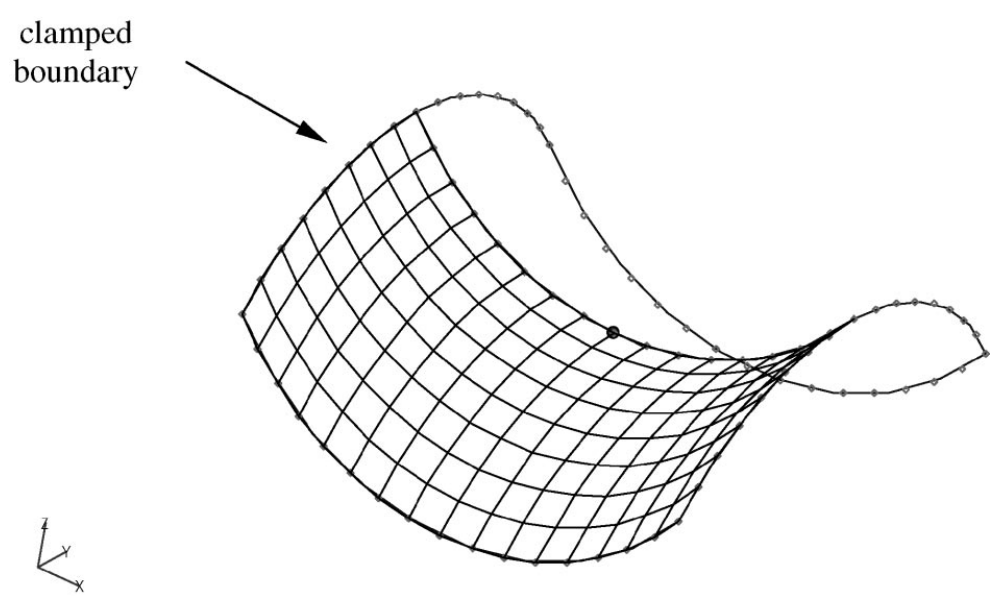

Figure 11. Partly clamped hyperbolic paraboloid. One half of the structure meshed with $16 \times 8$ four-node elements.

\subsection{Bending-dominated test I - Partly clamped hyperbolic paraboloid}

In analysing the asymptotic behaviour of shell elements as the thickness tends to zero, it is possible to define problems as being membrane or bending dominated. When this distinction is not clear the asymptotic deformation state is said to pertain to an intermediate state [26].

Following the analysis of Malinen and Pitkäranta [30], bending-dominated problems are known to be hard tests for standard finite element formulations, which provide a poor approximation for inextensional displacements, with numerical failure likely to occur.

A particular interesting bending-dominated test problem was introduced in the work of Chapelle and Bathe [27] and further developed in Reference [28]. The problem consists of a hyperbolic paraboloid shell structure, loaded by self-weight and clamped along one side, as represented in Figure 11. The geometric, material and load data are the adopted in Reference [28], and only one half of the surface needs to be considered in the analysis. 


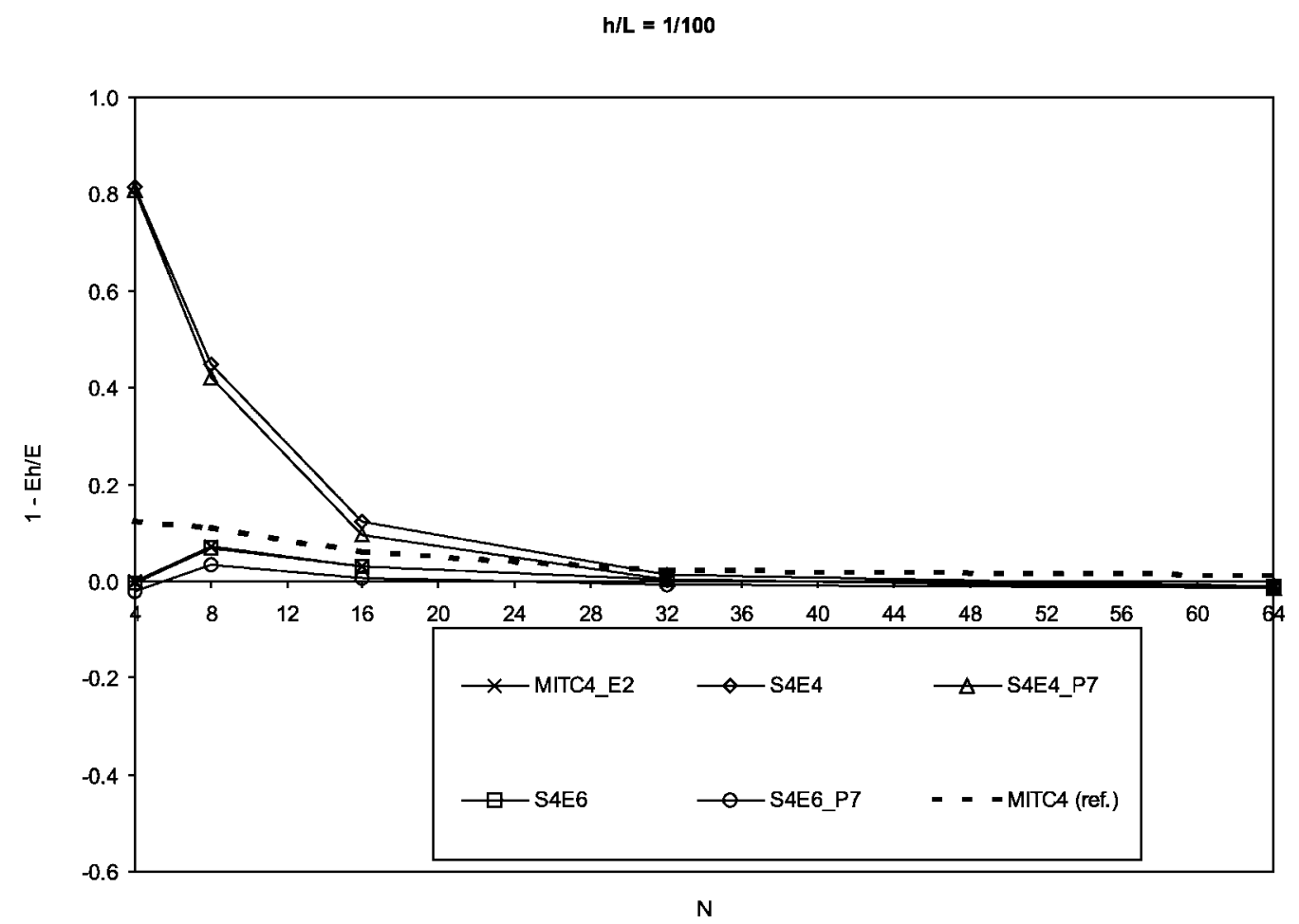

Figure 12. Convergence in strain energy for the hyperbolic paraboloid problem: $h / L=1 / 100$.

For the finite element mesh, sequences of $N \times N / 2$ elements $(N=4,8,16,32,64)$, where $N$ is the subdivisions along the $X$-axis, were adopted. Following the proposal of Bathe et al. [28], thickness-length $(h / L)$ relations of $1 / 100,1 / 1000$ and $1 / 10000$ were considered.

For this problem there is no analytical solution, and reference values for the total strain energy, previously obtained by Bathe et al. with a refined mesh of high-order shell elements, were used [28]. Graphically, we represent the strain energy error of the finite element solution in comparison to that reference value, i.e.

$$
E_{\mathrm{r}}=\frac{E-E_{\mathrm{h}}}{E}=1-\frac{E_{\mathrm{h}}}{E}
$$

with $E_{\mathrm{h}}$ being the strain energy of the finite element approximation and $E$ being its reference value.

The performance of the proposed elements can be verified in Figures 12-14, according to the thickness values adopted. The results of MITC4 element, as presented in [28], are also reproduced for comparison.

From the figures, it can be seen that the proposed elements performed quite well when compared with the MITC4 element. However, elements S4E4 and S4E4_P7 revealed a strong sensitivity to the decrease in thickness. For the ratio $h / L=1 / 1000$ and with the maximum mesh refinement allowed $(N=64)$ the convergence error is about 10 per cent, reaching unacceptable values for the relation $h / L=10000$. 


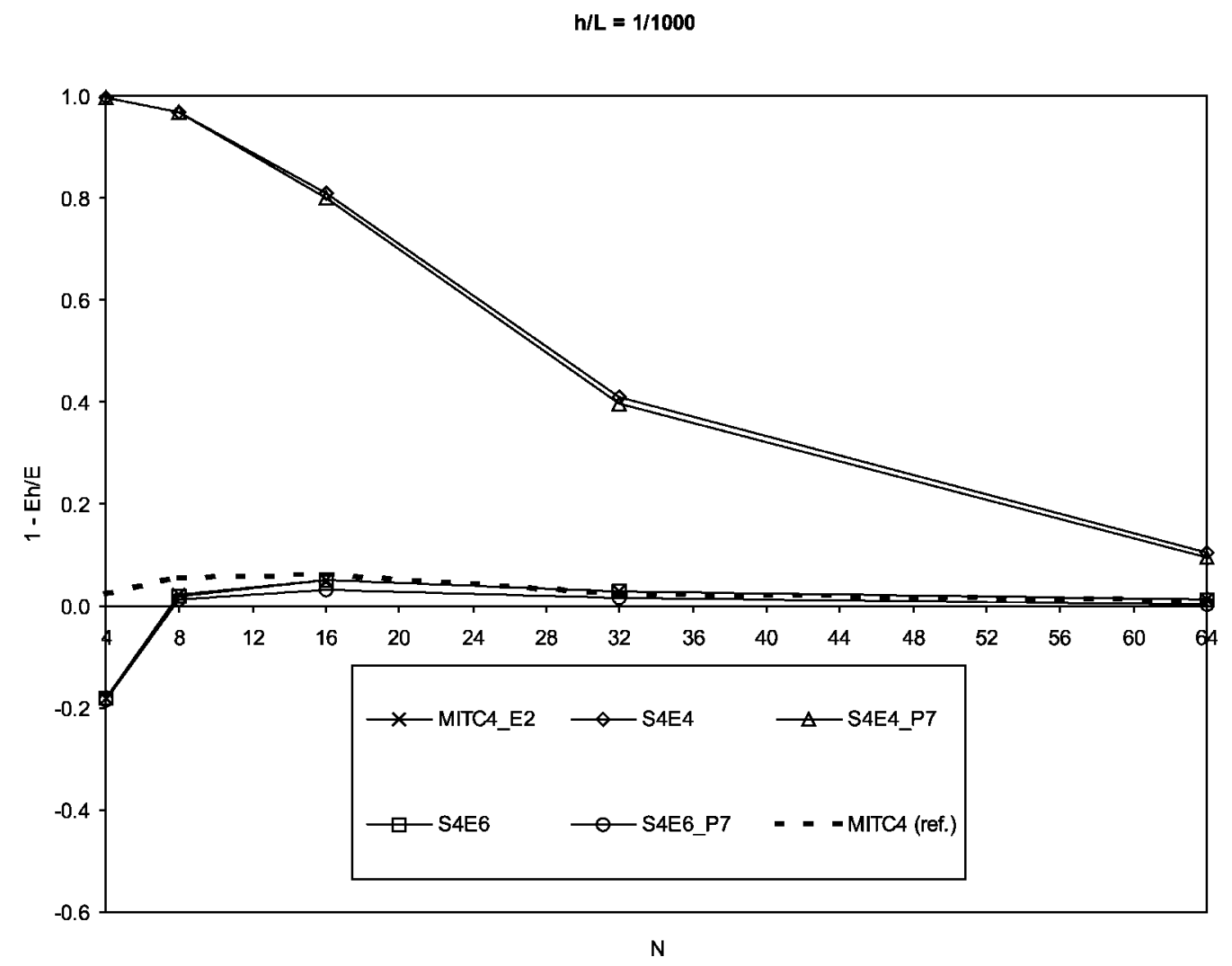

Figure 13. Convergence in strain energy for the hyperbolic paraboloid problem: $h / L=1 / 1000$.

In contrast the shell element S4E6_P7 obtained the best results for the ratio $h / L=100$, with elements S4E6 and MITC4_E2 showing similar results. For ratios $h / L$ of $1 / 1000$ and $1 / 10000$ these elements revealed an excess of flexibility with the lower refinement meshes $(N=4$ and 8$)$, showing however an improved behaviour as the mesh is refined.

\subsection{Bending-dominated test II - Free cylindrical shell}

In this example, a cylindrical shell of uniform thickness $h$, length $2 L$ and radius $R$ is loaded by an axially constant pressure distribution. The pressure varies with the angular distance from the apex (see Figure 1 in Reference [28]) following the function

$$
p(\varphi)=P_{0} \cos (2 \varphi)
$$

where the amplitude factor $P_{0}$ is scaled with the thickness as

$$
P_{0}=p_{0} h^{3}
$$

being $p_{0}$ a constant. 


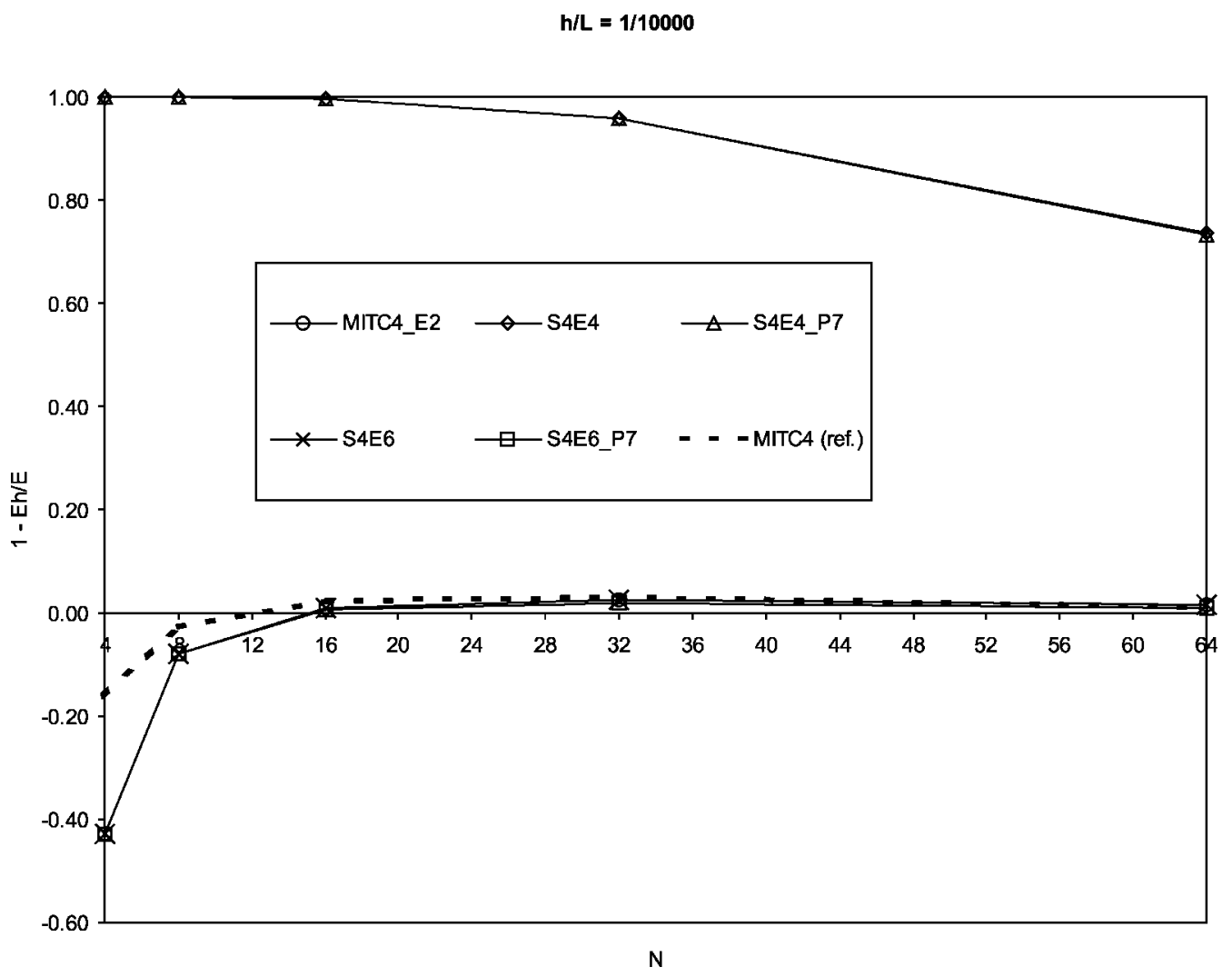

Figure 14. Convergence in strain energy for the hyperbolic paraboloid problem: $h / L=1 / 10000$.

The cylinder possesses free ends with no kinematical constraints, being self-supported by the load pattern. The geometry, material and load data are the ones provided by the study of Bathe et al. in Reference [28].

This problem has been extensively described by the group of Juhani Pitkäranta, with the publication of an analytical locking insight for various shell models early in 1995 [26]. The reference solutions for the test case are the ones published in the paper of Malinen and Pitkäranta [30].

In this simulation, $1 / 8$ of the structure were modelled by uniform mesh patterns of $N \times 2 N$ elements, being $N(N=4,8,16,32)$ the number of elements along the axial direction. As for the previous example, thickness-to-length ratios of $1 / 100,1 / 1000$ and 1/10 000 were employed, and a strain energy analysis was carried out with the results presented in Figures 15-17.

In this example all the proposed elements are performed in a very similar way. For the thickness-length ratio of $1 / 100$ the MITC4 element behaviour is clearly superior, as for the most refined mesh $(32 \times 64$ elements $)$ the proposed elements presents an error of 1.0 per cent, while Dvorkin and Bathe element leads almost to the correct solution. However, as thickness values decrease, all elements tend to converge to the same performance. It is particularly interesting to note that even the 4 enhanced parameters elements S4E4 and S4E4_P7, in 


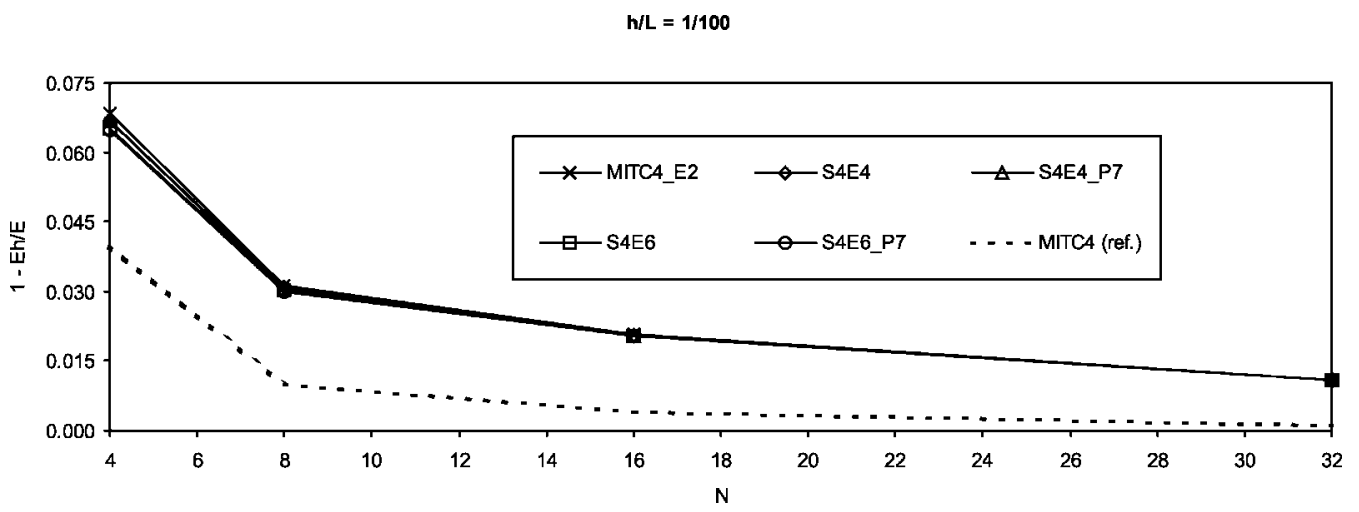

Figure 15. Convergence in strain energy for the free cylinder problem: $h / L=1 / 100$.

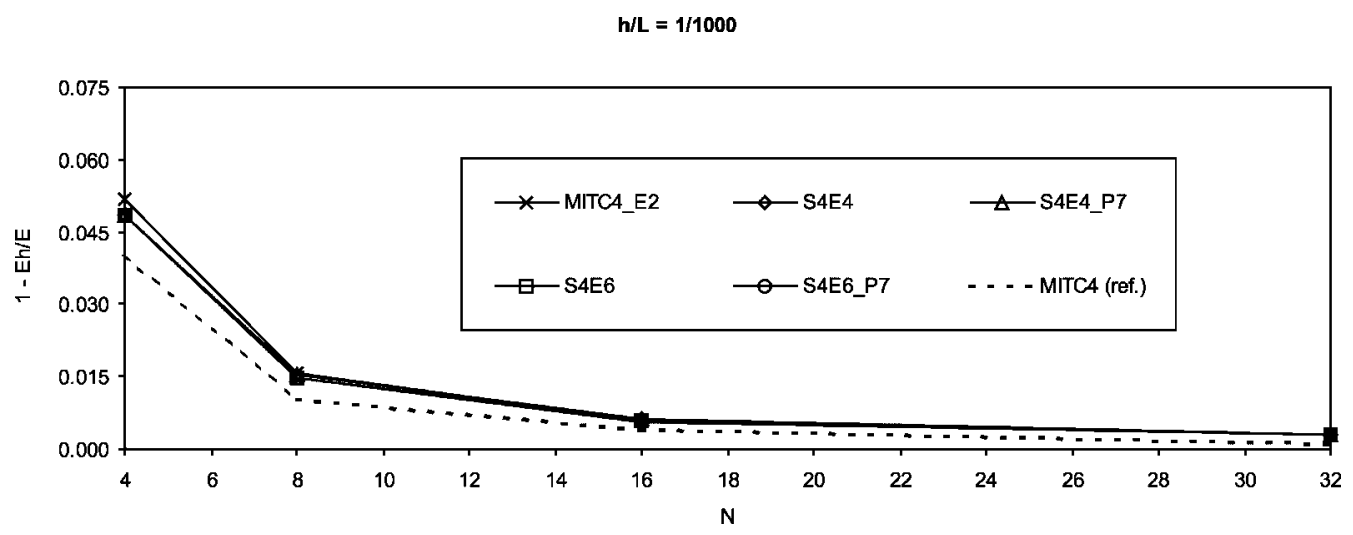

Figure 16. Convergence in strain energy for the free cylinder problem: $h / L=1 / 1000$.

opposition with the previous example, converged to a correct value for the lower thickness values.

\subsection{Membrane-dominated test I - Clamped cylindrical shell}

The geometry and loading for this example are the same as for the previous one, but now the cylinder presents clamped ends. This modification leads to a membrane-dominated problem, where pure bending is inhibited but still conducting to a well-posed problem (References [26-29]).

Although the load pattern remains the same, it is scaled in a way consistent of the membrane characteristic of the asymptotic behaviour. Doing so, Equation (38) is replaced by

$$
P_{0}=p_{0} h
$$

that is, the load amplitude now being a linear function of thickness values. 


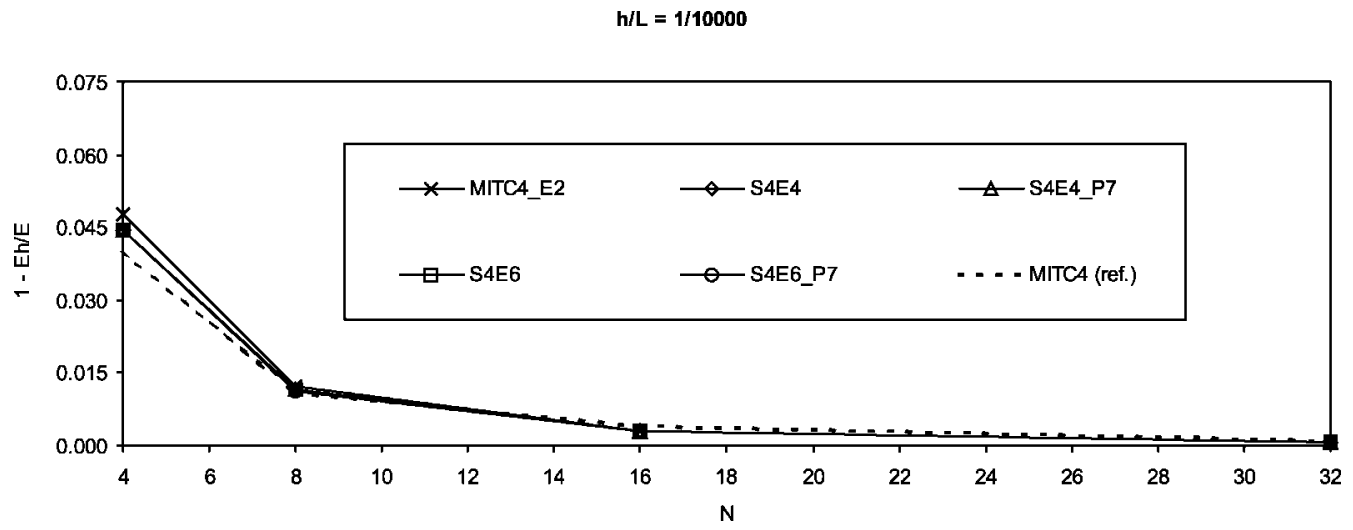

Figure 17. Convergence in strain energy for the free cylinder problem: $h / L=1 / 10000$.

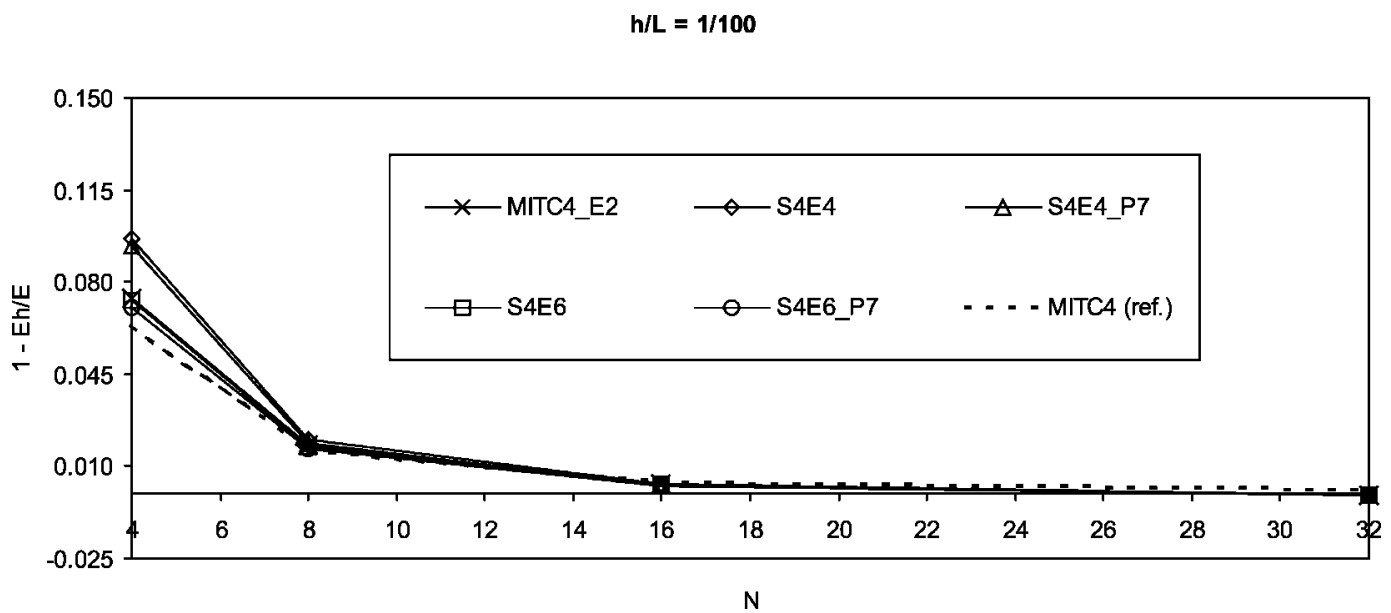

Figure 18. Convergence in strain energy for the clamped cylinder problem: $h / L=1 / 100$.

Using the same mesh configuration as for the free cylinder problem before, the strain energy is evaluated for every element presented and for the ratios thickness to length described before. Reference solutions come from Pitkäranta et al. [26, 30] and MITC4 results, as presented by Bathe et al. [28], are reproduced and the final results are indicated in Figures 18-20.

As thickness values tend to zero, the strain energy error of all elements increases. Nevertheless elements MITC4_E2, S4E6 and S4E6_P7 present similar results as Dvorkin and Bathe element. For the coarser mesh $(N=4)$ MITC4 element provides the best performance with differences between this element and the proposed ones vanishing as refinement is increased. It is also noticeable the lower convergence rate of S4E4 and S4E4_P7 elements. 


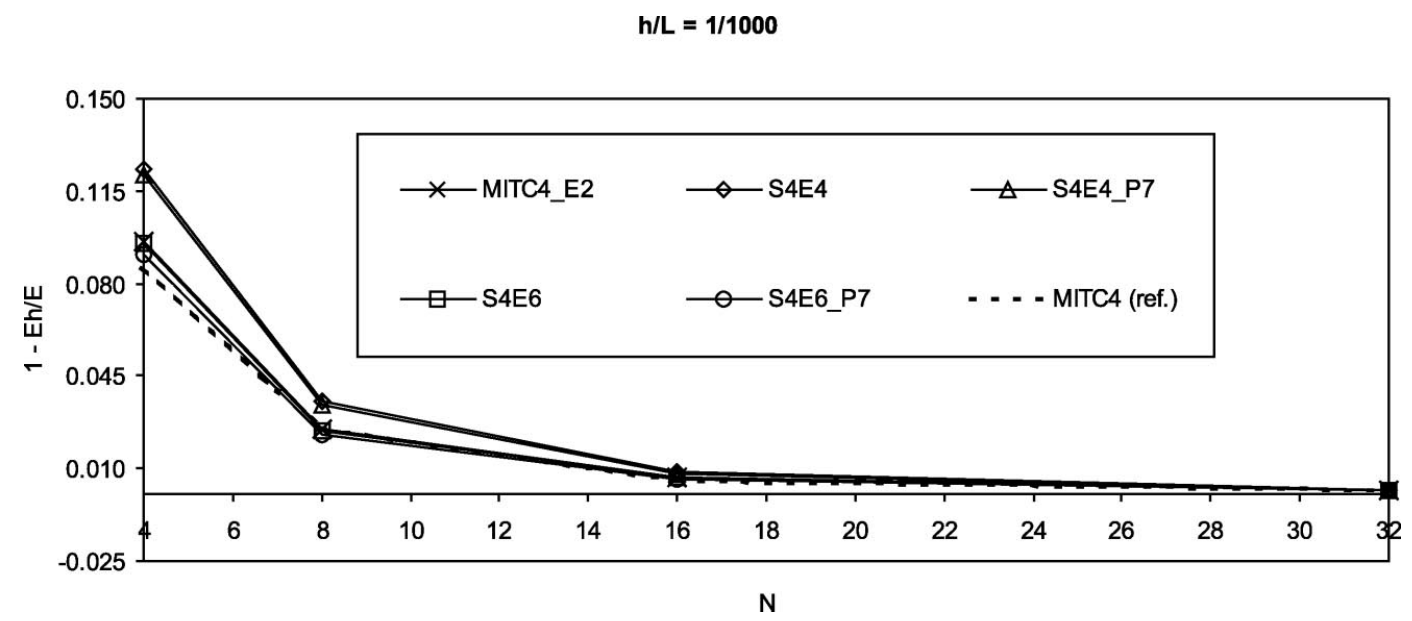

Figure 19. Convergence in strain energy for the clamped cylinder problem: $h / L=1 / 1000$.

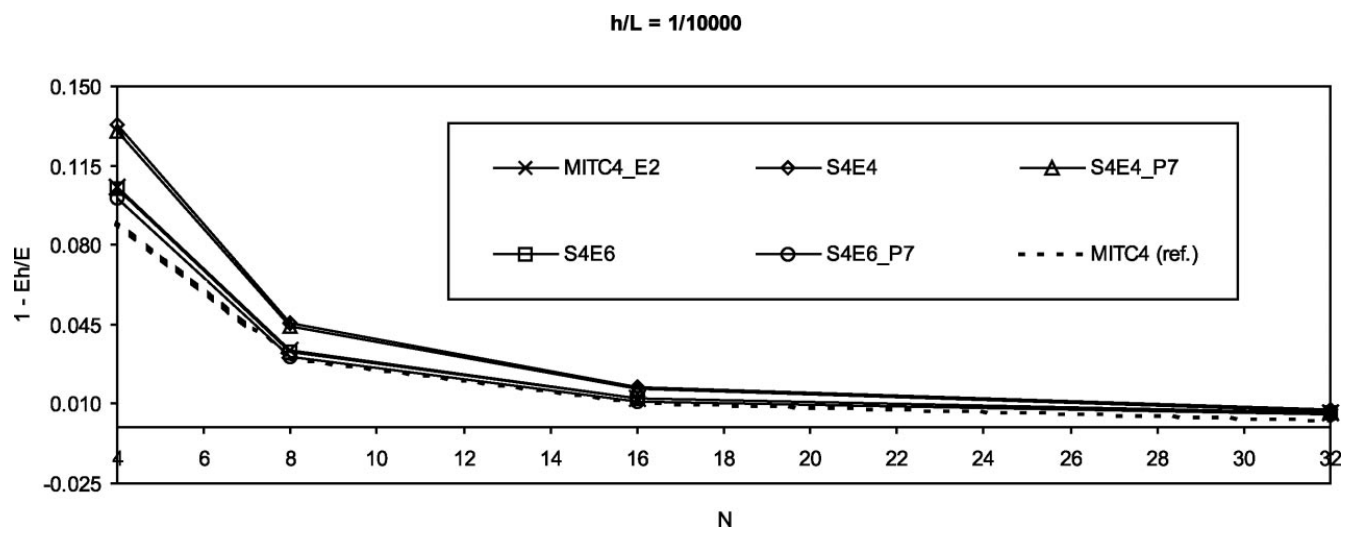

Figure 20. Convergence in strain energy for the clamped cylinder problem: $h / L=1 / 10000$.

\subsection{Membrane-dominated test II - Clamped hemispherical cap}

This benchmark, proposed by Chapelle and Bathe in [27], represents a hemisphere of radius $R$ and uniform thickness $h$ which is fully clamped and subjected to an axisymmetric pressure distribution on its outer surface (see Figure 11 in the previous reference). The imposed load is the same as the cylinder problems before, with the amplitude being scaled linearly with the thickness.

For the numerical simulation, the pertained data values are those from Reference [28], including mesh parameters such as the number of divisions in each of the directions of the structure. As usual with spherical type structures, a demonstrative partition of the shell is divided into 3 macro-surfaces, each of one being modelled in a mapped way, as can be seen in the work of Bathe et al. [28]. The results of Dvorkin and Bathe element MITC4 


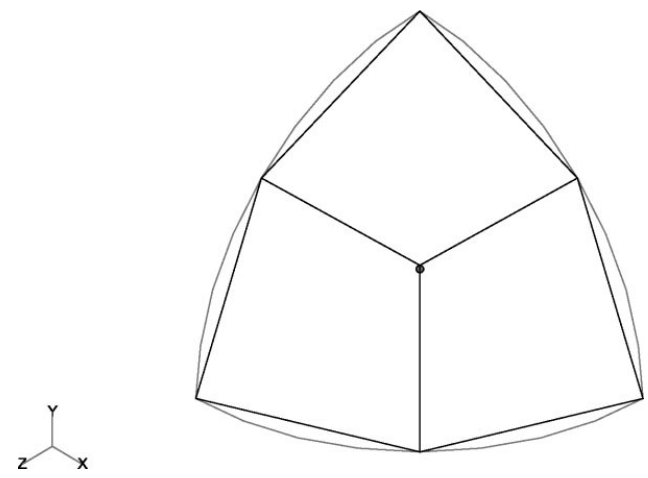

Figure 21. A $2 \times 8$ elements mesh for the hemispherical cap problem (only $1 / 4$ of the total structure is represented).

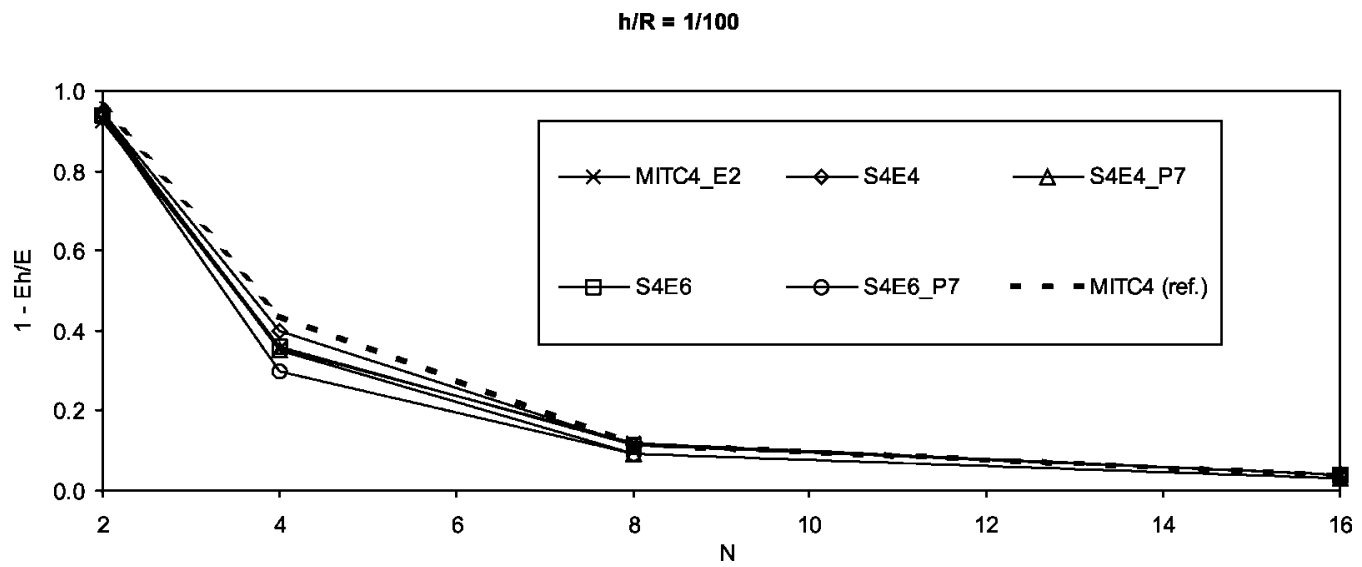

Figure 22. Convergence in strain energy for the clamped hemispherical cap problem: $h / R=1 / 100$.

are reproduced for comparison from the same reference, and the range of thickness to radius adopted are the same as the previous examples $(h / R=1 / 100,1 / 1000,1 / 10000)$.

This test problem is well suited for assessing distortion sensitivity levels on bilinear finite elements, once the modelled surface is only a crude representation of the correct model. For instance, in Figure 21 a partition (1/4) of the coarsest mesh (with a total of $2 \times 8$ elements) is represented, and the deficiencies in the correct representation of the hemispherical cap surface are clear.

This problem possess no available analytical solution, therefore reference values are taken from [28], where a 1D axisymmetric shell finite element model was employed. The comparative results between the present elements and Dvorkin and Bathe MITC4 elements are shown in Figures 22-24, where $N$ stands for the number of elements along the latitude direction.

From the obtained results it is clear that the proposed elements present good results, although all the formulations reveal slow convergence behaviour, with element S4E6_P7 showing the best results among the proposed elements. 


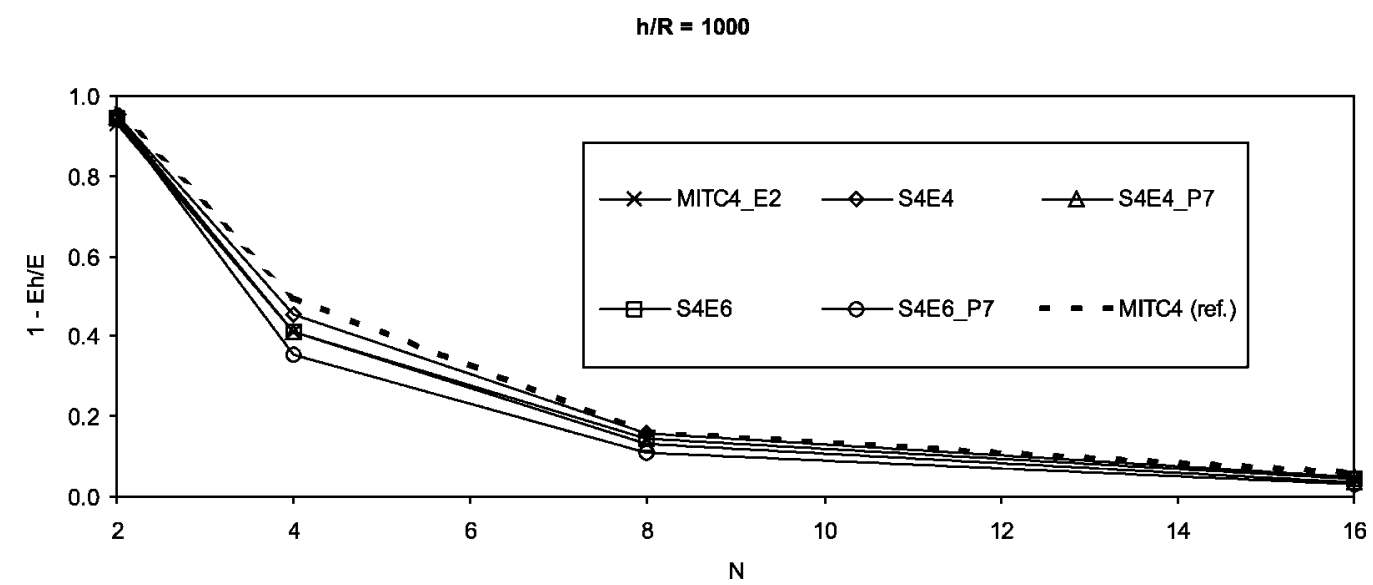

Figure 23. Convergence in strain energy for the clamped hemispherical cap problem: $h / R=1 / 1000$.

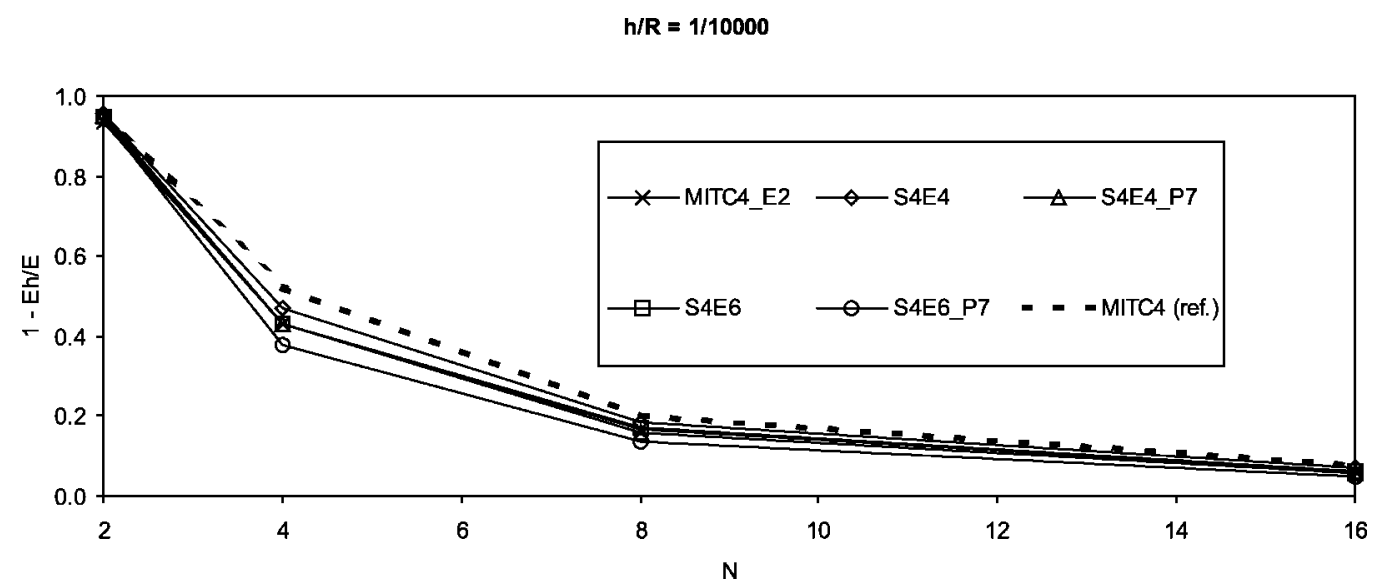

Figure 24. Convergence in strain energy for the clamped hemispherical cap problem: $h / R=1 / 10000$.

\section{CONCLUDING REMARKS}

A new insight into the transverse shear locking phenomenon affecting four-node degenerated shell elements was presented in this paper. Locking effects were related to the inability of the basis of the null transverse shear strain subspace, implicitly defined in the finite element formulations, to reproduce the required displacement field.

The proposal of the present work was to enlarge this subspace via the application of the enhanced assumed method directly over the transverse shear strain field. This approach conducted to a new class of four-node elements, with an improved performance when compared to the original degenerated element. 
The proposed elements differ with respect to the number of internal variables used and also relating to the inclusion (or not) of an additional in-plane enhancement. The transverse shear strain subspace analysis also led to the improvement of the MITC4 element, originally based on the assumed natural strain approach, and now also enriched with enhanced modes.

The elements proposed were subjected to linear numerical test problems, being compared to results reported in the literature for the well-known shell element formulations. The performance of the S4E6 and S4E6_P7 elements revealed a strong improvement when compared to the classical degenerated approach, and also in some examples when compared to other well-known formulations. The S4E4 and S4E4_P7 performance was not always superior and, therefore, these elements should not be considered reliable in a general sense. The MITC4_E2 element also performed well, namely in the Morley skew plate, with better results than elements based on formulations using membrane-based enhancements. The extension of the presented formulation into non-linear cases is being performed.

\section{ACKNOWLEDGEMENTS}

The work of Robertt A. Fontes Valente and Pedro M. Almeida Areias were supported by 'Ministério da Ciência e da Tecnologia, Fundação para a Ciência e a Tecnologia', Portugal, under grants PRAXIS $\mathrm{XXI} / \mathrm{BD} / 21662 / 99$ and PRAXIS XXI/BD/18533/98, respectively. This work is also included in the project from 'Fundação para a Ciência e a Tecnologia' and FEDER through the grant POCTI/33640/ EME/2000. These supports are gratefully acknowledged.

\section{REFERENCES}

1. Ahmad S, Irons BM, Zienkiewicz OC. Analysis of thick and thin shell structures by curved finite elements. International Journal for Numerical Methods in Engineering 1970; 2:419-451.

2. Bucalem ML, Bathe KJ. Finite element analysis of shell structures. Archives of Computational Methods in Engineering 1997; 4(1):3-61.

3. Yang HTY, Saigal S, Masud A, Kapania RK. A survey of recent shell finite elements. International Journal for Numerical Methods in Engineering 2000; 47:101-127.

4. Kui LX, Liu GQ, Zienkiewicz OC. A generalized displacement method for the finite element analysis of thin shells. International Journal for Numerical Methods in Engineering 1985; 21:2145-2155.

5. Andelfinger U, Ramm E. EAS-Elements for two-dimensional, three-dimensional, plate and shell structures and their equivalence to HR-elements. International Journal for Numerical Methods in Engineering 1993; 36:1311-1337.

6. Zienkiewicz OC, Taylor RL, Too JM. Reduced integration techniques in general analysis of plates and shells. International Journal for Numerical Methods in Engineering 1979; 3:275-290.

7. Hughes TJR, Cohen M, Haroun M. Reduced and selective integration techniques in finite element analysis of plates. Nuclear Engineering and Design 1978; 46:203-222.

8. Belytschko T, Tsay C. A stabilization procedure for the quadrilateral plate element with one-point quadrature. International Journal for Numerical Methods in Engineering 1983; 19:405-419.

9. Militello C, Felippa C. A variational justification of the assumed natural strain formulation of finite elements, Part I: Variational principles. Computers and Structures 1990; 34(3):431-438.

10. Dvorkin EN, Bathe KJ. A continuum mechanics based four-node shell element for general nonlinear analysis. Engineering Computations 1984; 1:77-88.

11. MacNeal RH, Derivation of element stiffness matrices by assumed strain distributions. Journal of Nuclear Engineering Design 1982; 70:3-12.

12. Bathe KJ, Dvorkin EN. A four-node plate bending element based on Mindlin/Reissner plate theory and a mixed interpolation. International Journal for Numerical Methods in Engineering 1985; 21:367-383.

13. Huang HC, Hinton E. A new nine node degenerated shell element with enhanced membrane and shear interpolation. International Journal for Numerical Methods in Engineering 1986; 22:73-92.

14. Bathe KJ, Dvorkin EN. A formulation of general shell elements - the use of mixed interpolation of tensorial components. International Journal for Numerical Methods in Engineering 1986; 22:697-722. 
15. Bucalem ML, Bathe KJ. Higher-order MITC general shell elements. International Journal for Numerical Methods in Engineering 1993; 36:3729-3754.

16. Simo JC, Rifai MS. A class of mixed assumed strain methods and the method of incompatible modes. International Journal for Numerical Methods in Engineering 1990; 29:1595-1638.

17. Büchter N, Ramm E, Roehl D. Three-dimensional extension of non-linear shell formulation based on the enhanced assumed strain concept. International Journal for Numerical Methods in Engineering 1994; 37:2551-2568.

18. Bischoff M, Ramm E. Shear Deformable shell elements for large strains and rotations. International Journal for Numerical Methods in Engineering 1997; 40:4427-4449.

19. Huettel C, Matzenmiller A. Consistent discretization of thickness strains in thin shells including 3D-material models. Communications in Numerical Methods in Engineering 1999; 15:283-293.

20. Yunhua L, Eriksson A. An alternative assumed strain method. Computer Methods in Applied Mechanics and Engineering 1999; 178:23-37.

21. Stander N, Matzenmiller A, Ramm E. An assessment of assumed strain methods in finite rotation shell analysis. Engineering Computations 1989; 6:58-66.

22. Roehl D, Ramm E. Large elasto-plastic finite element analysis of solids and shells with the enhanced assumed strain concept. International Journal of Solids and Structures 1996; 33:3215-3237.

23. Eckstein A, Basar Y. Ductile damage analysis of elasto-plastic shells at large inelastic strains. International Journal for Numerical Methods in Engineering 2000; 47:1663-1687.

24. César de Sá JMA, Owen DRJ. The imposition of the incompressibility constraint in finite elements-a review of methods with a new insight to the locking phenomenon. In Proceedings of the 3rd International Conference on Numerical Methods for Non-Linear Problems, Taylor C et al. (eds). Dubrovnik, Pineridge Press Ltd: Swansea, U.K., 1986.

25. César de Sá JMA, Natal Jorge RM. New enhanced strain elements for incompressible problems. International Journal for Numerical Methods in Engineering 1999; 44:229-248.

26. Pitkäranta J, Leino Y, Ovaskainen O, Piila J. Shell deformation states and the finite element method: a benchmark study of cylindrical shells. Computer Methods in Applied Mechanics and Engineering 1995; 128:81-121.

27. Chapelle D, Bathe KJ. Fundamental considerations for the finite element analysis of shell structures. Computers and Structures 1998; 66:19-36.

28. Bathe KJ, Iosilevich A, Chapelle D. An evaluation of the MITC shell elements. Computers and Structures 2000; 75:1-30.

29. Chapelle D, Bathe KJ. The mathematical shell model underlying general shell elements. International Journal for Numerical Methods in Engineering 2000; 48:289-313.

30. Malinen M, Pitkäranta J. A benchmark study of reduced-strain shell finite elements: quadratic schemes. International Journal for Numerical Methods in Engineering 2000; 48:1637-1671.

31. Bathe KJ. Finite Element Procedures (2nd edn). Prentice-Hall: Englewood Cliffs, NJ, 1996.

32. Huang HC. Static and Dynamic Analyses of Plates and Shells-Theory, Software and Applications. Springer: Berlin, Heidelberg, 1989.

33. César de Sá JMA. Numerical modelling of incompressible problems in glass forming and rubber technology. Ph.D. Thesis, C/Ph/91/86, University College of Swansea, University of Wales, U.K., 1986.

34. Morley LSD. Skew Plates and Structures. Pergamon Press: Oxford, 1963.

35. Belytschko T, Stolarski H, Liu WK, Carpenter N, Ong JSJ. Stress projection for membrane and shear locking in shell finite elements. Computer Methods in Applied Mechanics and Engineering 1985; 51:221-258.

36. Scordelis AC, Lo KS. Computer analysis of cylindrical shells. Journal of American Concrete Institute 1969; 61:539-561. 